\title{
Crosby Arboretum (Picayune, Mississippi): A Natural World for All
}

\author{
Banu Ozturk Kurtaslan and Robert Brzuszek
}

Additional information is available at the end of the chapter

http://dx.doi.org/10.5772/55815

"At the Crosby Arboretum, Picayune, Mississippi, I listened for and found my voice..." Edward L. Blake Jr.

\section{Introduction}

Arboretums are living plant museums where mainly the taxa of trees and other wood-like plants of known origin and age, each of which are gathered in a correct and careful manner for the purposes of scientific research and observation, are cultivated, exhibited and introduced in suitable selected habitats [1].

Arboretums are garden abstractions for interpreting the natural landscape. These gardens offer biological and ecological diversity of collections, serve as conservation of soil, water and biological resources, demonstration of environmentally responsible landscape design, and restoration of degraded landscapes.

Being of vital importance for the protection of the nature in the present day urban life, arboretums at the same time have important roles in terms of esthetical and recreational aspects. In addition to providing scientific data regarding plants, arboretums also assume the function of presenting their natural beauties, esthetical qualities (size, form, texture, line and color characteristics) and introducing rich species of plants [2].

The functions of arboretums, whose formation on the earth dates back centuries ago, shows variety. These functions are giving information to all the students from elementary and secondary education to the university level and to the local community primarily about wood-like plants and secondly if they desire, about herbaceous plants, introducing these plants in their habitats and contributing to the development of a consciousness of protecting the environment. Besides, other functions of arboretums can be listed as introducing the natural, endemic and exotic plants from all corners of the world without having to go on 
long and expensive trips through gathering them as climate allows, giving people the opportunity to select the plants with esthetical value, taking endangered species under preservation, conducting studies on the adaptation of the species of foreign origin to the country. Furthermore, the functions of special-purpose arboretums will certainly be different. Arboretums that are organized to maintain the cultivation of a limited number of species can be given as an example to such places [3].

In the beginning, botanical gardens and arboretums were generally created for the introduction and production of newly discovered plants. Big botanical gardens, such as Arnold Arboretum or Kew Botanical Garden in London, collect plant species from all around the world and maintain the affluence of these species by developing and reproducing them. In recent times, this function has become of secondary importance. This is because the number of newly discovered plants is not as much as it was in the 19th century. However, there are still travels for discovering new species [4].

The functions of arboretums can be summarized as follows:

- They maintain the physical balance of the city. They create buffer areas in cities by eliminating the disharmonies and mutual negative effects among different areas of use such as housing, commerce and industry,

- They have positive effects on human psychology through providing an opportunity for recreation,

- They are the oxygen sources of cities,

- They bring in positive microclimatic characteristics to the city. Wide green areas considerably improve the air circulation in cities, provide clean air to the city and they are effective in increasing atmospheric moisture. It was determined that the temperature in open and green areas was 6.5 degrees cooler in winter and 10 degrees cooler in summer compared to the temperature in the city. Green areas humidify the air through transpiration, thus the dust in the humid air either settles as suspended particles or are taken away through the wind [4].

It is a necessity that arboretums are planned and designed through an ecological approach in order that they can perform their assumed ecological functions. In landscape architecture, "ecological design" is one of the significant aplication of sustainibility. According to Van der Ryn and Cowan (1996) ecological design is "any form of design that minimizes environmentally destructive impacts by integrating itself with living processes." Ecological design can apply to many design systems such as architecture, agriculture, engineering and many other fields. In the scope of this chapter, which is about an arboretum, ecological design is an important concept in terms of "sustainable landscape design" [5] [6] [7].

In the XIX century ecological awarenes was reflected to modern urban landscape design and planning by theorists and practitioners. For instance Frederic Law Olmsted produced projects such as Central Park (New York), Emerald Necklace (Boston) and other national parks, and was a pioneer of landscape architecture. This ecological sensitivity continued with Jens Jensen, Ian Mc Harg, Anne Winston Spirn and Michael Hough [8] [9]. 
An example of early ecological design and planning, "Crosby Arboretum is one of the substantial ecological design works in terms of contributions on the urban/nature context and the development of natural settings in cities. It was established in 1978 as a living memorial to L.O. Crosby Jr., a prominent Mississippi timber owner and philantropist. The idea of his immediate family was to create an arboretum and form a board of directors to fully explore the concept of ecological design. The Crosby family donated a 26-ha (64-acre) site on which to establish the arboretum. The primarily wetland site was formerly a working strawberry farm in the 1940s, complete with drainage ditches and old farm roads, that had been converted to a pine plantation shortly thereafter " [10].

The arboretum purchased and leased nearby lands containing pristine examples of local native plant communities, realizing that nearby natural plant communities would become important research models for the exhibit design of the arboretum, as well as to fulfill the preservation aspect of their mission statement. So it was designed by abstracting the natural habitats of plants and animals, within the Pearl River Basin. The Pearl River is a 444-mile long major watershed in west Mississippi that Arboretum lands lie within. Each exhibit illustrates a combination of past human influences and natural succession that reveals the drama and beauty of the regional flora. During a 10-y period, 11 sites comprising more than $685 \mathrm{ha}(1700 \mathrm{ac})$ would become the satellite natural areas of the arboretum, and they were systematically inventoried by biologists. Detailed topographical surveys were conducted on the mostly flat site, and subtle moisture gradients and drainage areas were recorded [10].

The mission of the arboretum is preserving, protecting, and displaying plants native to the Pearl River Drainage Basin ecosystem, providing environmental and botanical research opportunities, and offering cultural, scientific, and recreational programs [11].

The Crosby Arboretum is a not-for-profit institution dedicated to educating the public about their environment by:

- Preserving, protecting, and displaying plants native to the Pearl River Drainage Basin in Mississippi and Louisiana

- Providing environmental and horticultural research opportunities

- Offering cultural, educational, scientific, and recreational programs

(http://www.crosbyarboretum.msstate.edu/pages/mission.php)

The arboretum located in Picayune, Mississippi is administered by the Mississippi State University Extension (Figure 1). These lands provides habitats for 300 native plant species and animals, some of which are endangered or threatened.

With increasing value being placed on our natural heritage, The Crosby Arboretum is the premier native plant conservatory in the southeast United States. The Arboretum has expanded to become a resource for education in the region and the world. Today, it provides for the protection of the region's biological diversity and also a place for the public's enjoyment of plant species native to the Pearl River Drainage Basin of south-central Mississippi and Louisiana. People can study and learn about plants and plant products so that they may use them to their best advantage and ensure their continuous propagation in 


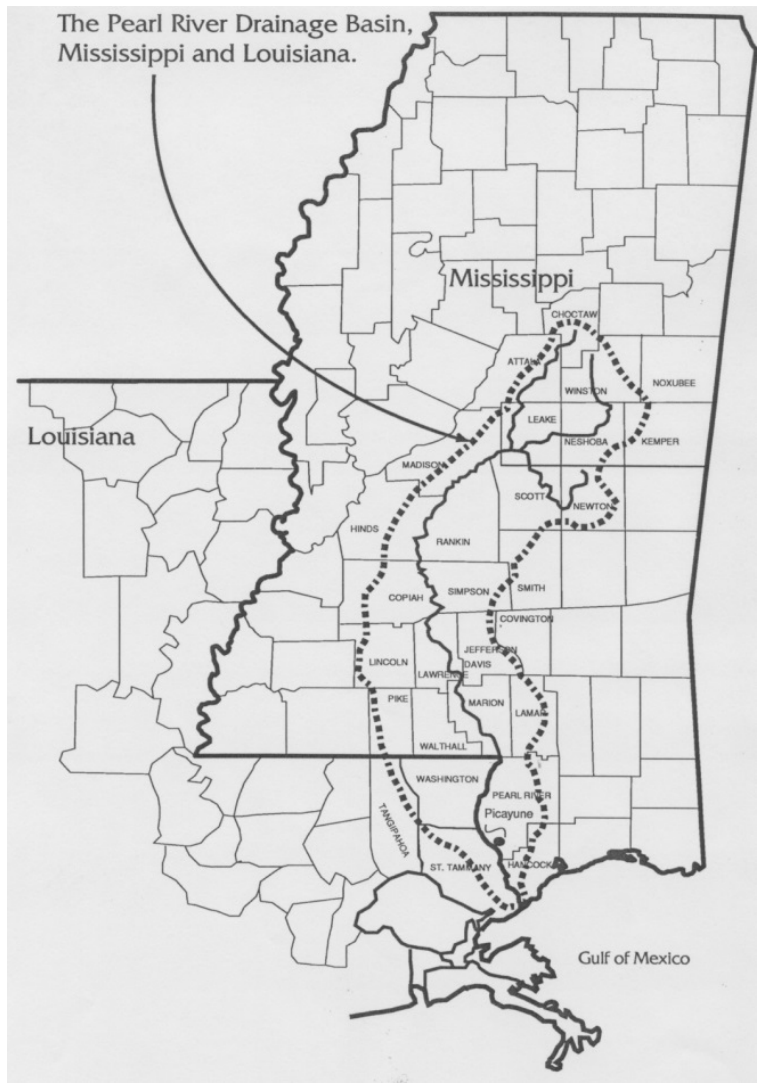

Figure 1. Location of the Crosby Arboretum.

the future. Aesthetic, agricultural, scientific, and industrial contributions of native plant species and ecosystems can be examined in a real-life setting at the Arboretum.

The arboretum has a 104-acre Native Plant Center and it serves as the focus of Arboretum activities and development. The Pinecote Pavilion and the Piney Woods Lake at the arboretum display native water plants in their natural setting. The Pinecote Pavilion and the many wooden bridges that complement the lake were designed by award-winning architect Fay Jones, of Fayetteville, Arkansas to enhance the artistic and functional aspects of the Arboretum.

(http://www.crosbyarboretum.msstate.edu/pages/about.php)

The Crosby Aboretum has 6000 visitors annually. Pinecote, the name of the Arboretum site, was designed to weave the activities and needs of human into an evolving living mosaic of "woodland, aquatic and pine savanna" habitats. This 64 acre former strawberry farm and pine plantation is being transformed to display and interpret the native plant communities found in our natural areas. 
The Arboretum was designed to be an evolving landscape that abstracts the natural habitats of plants and animals within the Pearl River Basin. Each exhibit illustrates a combination of past human influences and natural succession that reveals the drama and beauty of the regional flora. The pond, slough, pathways, bridges, landscape features and pavilion weave together into a seamless whole, fortifying each other's presence (Figure 2).

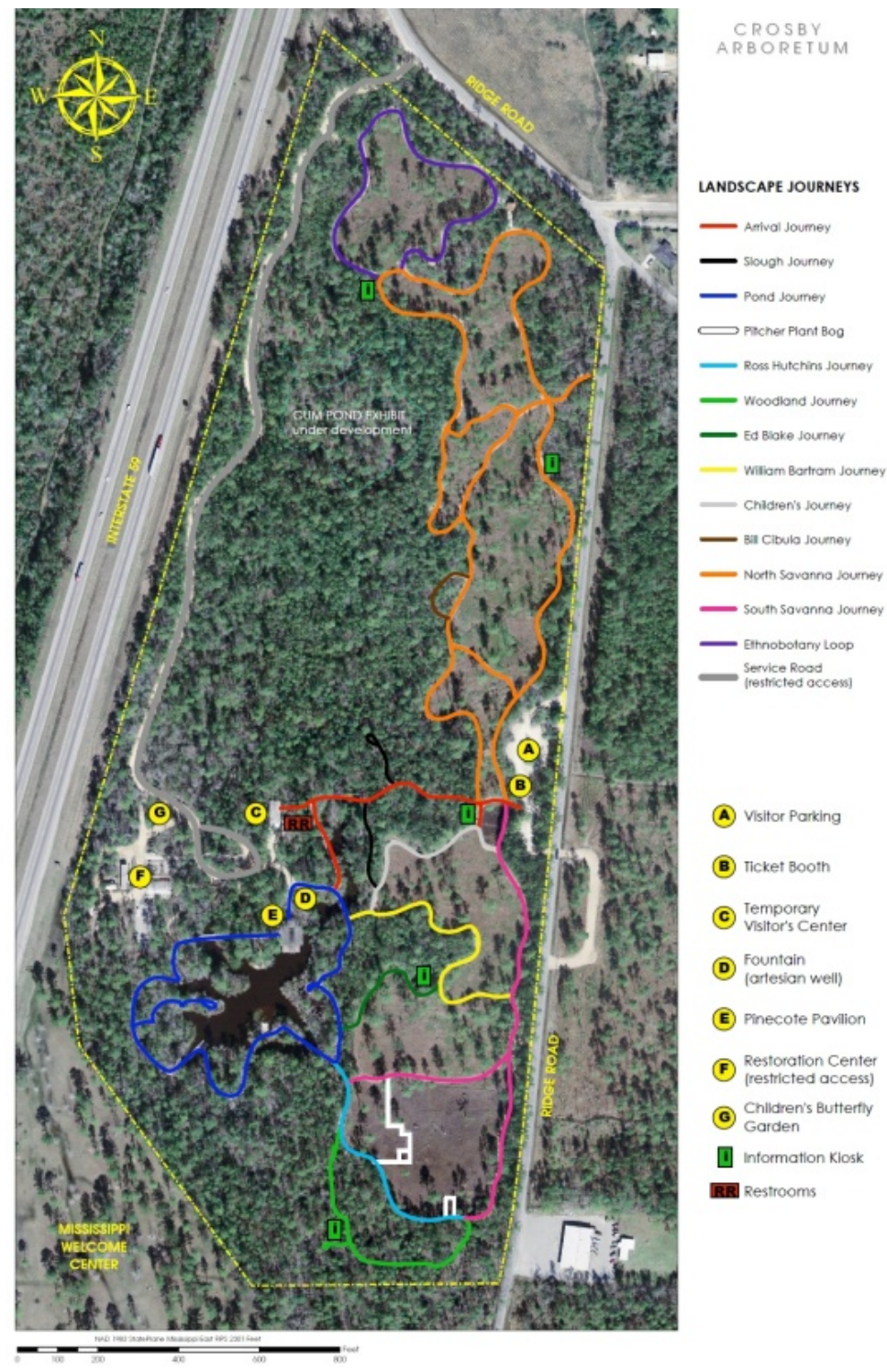

Figure 2. Crosby Arboretum trails and other uses (http://www.crosbyarboretum.msstate.edu/pages/map.php) 
The Crosby Arboretum Master Plan was completed in 1994. The Schematic Master Plan outlined the following design principles to the Arboretum's Interpretive Center (Pinecote) site development:

- Displaying habitats as well as flora

- Creating displays that enhance the character of the native landscape

- Fostering a sense of place appropriate to the Piney Woods region of Mississippi

- Interpreting how perceptions of landscape by industry, agriculture, and forestry have resulted in changes in landscape appearance and land use patterns

- Developing a holistic approach to interpreting what we see

- Encouraging a synthesis to develop between the arts, sciences, and humanities

- Focusing on doing a few things well and emphasizing quality

- Interpreting the role of fire as a major determinant of landscape form

- Displaying the arboretum landscape as a process rather than product

Andropogon Associates Ltd articulated the design at Pinecote as "a new synthesis of the artistic values of drama and beauty, with the scientific values of correct relationships between plant and plant and plant and place. This design is based on 3 premises:

1. The Pinecote site will be treated as an entire coherent unit.

2. The site design at Pinecote will accurately reflect the major natural processes of the site.

3. Planting design should reflect Plant Community Structure." [10].

"In spring 2011, a graduate class in the department of landscape architecture at Mississippi State University, was assigned by one of the authors (Brzuszek) the task of conducting the research and to develop the conceptual designs for the Forested Stream exhibit. The semester-long project included studying the research literature for small streams and stream restoration; site visits to small streams near the Arboretum site to measure and map their wetland configurations; study the plant species and spatial configurations of small stream corridors; to host a design charrette to consider possible conceptual designs; and the resolution of conceptual ideas into a proposed exhibit design. To fund the exhibit construction, a federal grant was applied for and awarded through the National Fish and Wildlife Foundation's 5-Star Grant. The grant awarded $\$ 38,870$ with a complementary match by the Crosby Arboretum and its partners, and the period required construction of the exhibit to be completed by June 2013" [12]. The small stream swamp exhibit covers approximately four acres and connects the Gum Pond in the northern part of the site to the Slough and Beaver Pond to the south. This wetland exhibit improves habitat for fish, amphibians, reptiles, and birds that are indigenous to the Pearl River Drainage Basin. The forested stream channel offers a unique opportunity to teach visitors about the importance of forested wetlands by demonstrating the value of its function [10]. Dead Tiger Creek, a natural stream which is near the arboretum, served as a reference site to closely examine the regional features of water corridors. The class studied vegetative patterns and aquatic habitats to aid in understanding the dynamics of small stream swamp forests. With collective effort, the class created field sketches and detailed notes on various characteristics of the creek. Reviewing this information provided vital intelligence and was relevant to future implementation of the Crosby Arboretum exhibit [13]. 


\section{The ecological significance of the Arboretum}

The Crosby Arboretum is preserving existing ecosystems in its natural areas and at the Interpretive Center site. [14][15] [16]. The designers enabled the site to "express itself" by introducing periodic fire to maintain the savanna exhibit (fire is an important component of the Piney Woods ecosystem) and it displays native plants within the context of regionally occurring native plant communities (Figure 3) [10] [17]. The arboretum is an environmental intervention and it is the first truly regenerative arboretum in the U.S. because the entire site, and all of its sites are based solely upon the environment [18] [19]. With these qualities, Crosby Arboretum is involved in four ecological landscape design categories which were determined by Mozingo (1997) [20]: "preservation of existing, functioning ecological systems; enhancement or re-establishment of degraded ecological systems; intensification of ecological processes to mitigate potential or existing ecological degradation; and environmental interventions which reduce nonrenewable resource consumption."

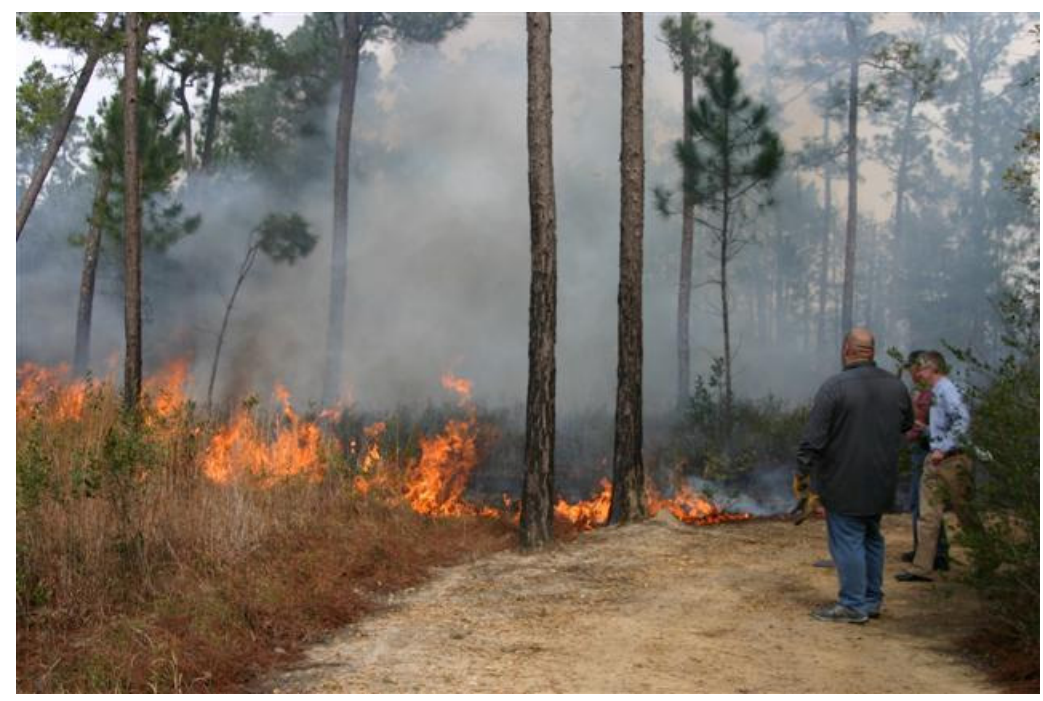

Figure 3. Precribed fires at te arboretum

The assemblages of carefully selected and protected lands nurtures the species of indigenous trees, shrubs, wildflowers, and grasses. Together with the rare, or endangered species of plants and wildlife throughout the Arboretum's preserves, unusual plants have their place as well. The Arboretum protects and manages several pitcher plant bogs both on site and within the natural areas. Edible, poisonous, and aromatic plants, too, are found at the Arboretum. As the seasons unfold their splendor, the Arboretum provides a clear, unobstructed view of the variety and beauty of our natural resources.

While constituting the master plan of the arboretum; through observation and research, first the staff uncovered the wet, mesic and dry zones of the site and their associated plants. This information guided the form of Pinecote's master plan (awarded an honour award by the 
American Society of Landscape Architects in 1992). At the Arboretum, each exhibit is modeled after natural habitats found and oriented at appropriate locations. Predominant plant species found in these habitats are then planted among the existing vegetation structure of Pinecote. These "introduced" species are located with scientific accuracy and designer's eye so that visitors have an opportunity to understand the process that shape plant communities as well as to experience the heightened drama and beauty of the Piney Woods.

The Pearl River Basin is at the hearth of the Arboretum's mission, which is to interpret, promote and preserve the native plant communities along its boundaries. Pinecote has no irrigation lines or other artificial life support systems, thus existing site hydrology plays a crucial role in the survival and management of all plant community exhibits. So from this standpoint, it paid attention to the water related designs in the arboretum constituting process. The Master Plan identifies the construction of four main wetland exhibits that are based upon regional water features. These include;

- a two-acre pond called Piney Woods Lake that abstracts the form and function of locallly-occurring beaver ponds;

- a half-acre Slough Exhibit based upon local bayous;

- a one acre Gum Pond exhibit that features a Gulf Coast waterbody primarily composed of tupelo gum trees (Nyssa sylvatica var. biflora), (Figure 4) and

- a 970' small stream corridor entitled the Forested Stream Exibit. The Piney Woods Lake, Slough, and Gum Pond exhibits have already been constructed prior to 2011.

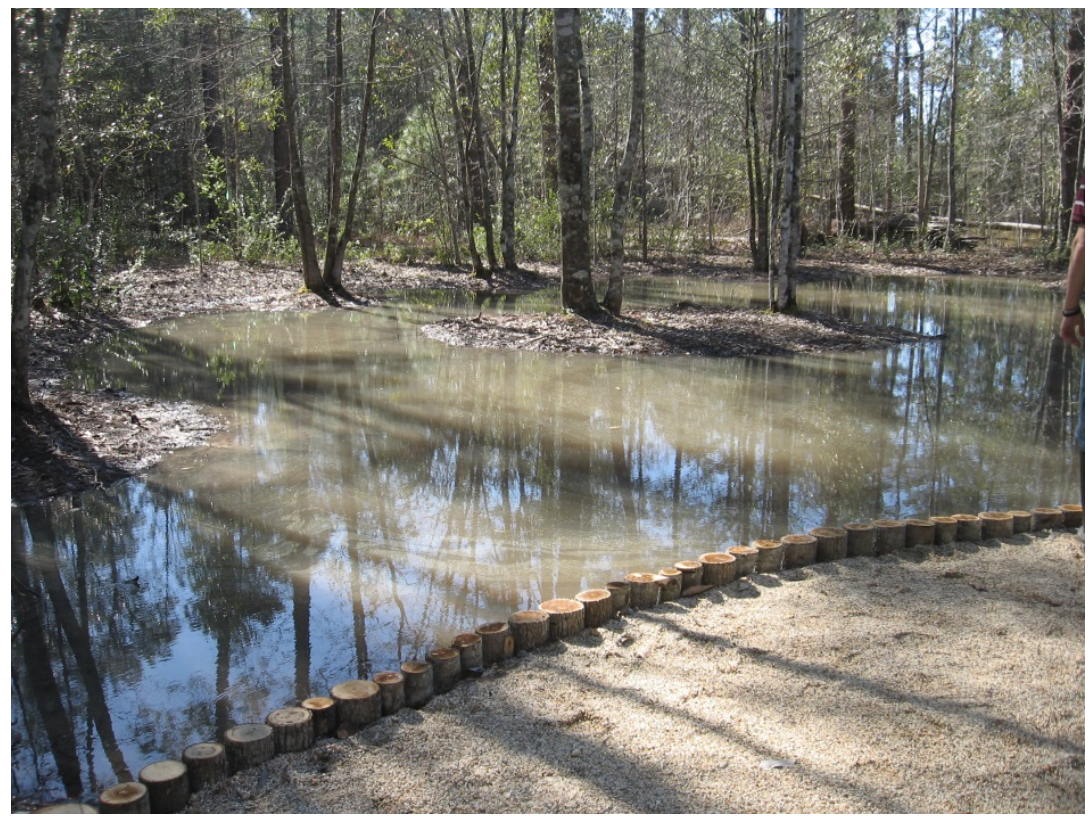

Figure 4. Gum Pond Exhibit area (Photo :Banu Ozturk Kurtaslan, 2011). 
After the completion of Beaver Pond, the success of the pond was demonstrated when beavers moved in and cut down every Taxodium tree that had been freshly planted along the pond's margin. Since then a fence has been constructed along the drainage outlet to reduce the number of problem animal species (Figure 5).

The Mississippi River system alone drains more than 40 percent of the United States and portions of southern Canada. The original plant community of Pinecote was essentially wetland in character, a wet pine savanna. Pinus palustris once dominated the dry rises of the site, supported with a understory of Aristida, Andropogon and a host of wild flowers. Even after row-cropping in the 1930s, mant native wetland plants such as Sarracenia alata, Ericaulon decangulare and Habenaria ciliaris reappeared at Pinecote, sustained by periodic fires set for forestry purposes. These wetland species continue to survive today and their locations form the basis for the design of Pinecote's plant displays [21].

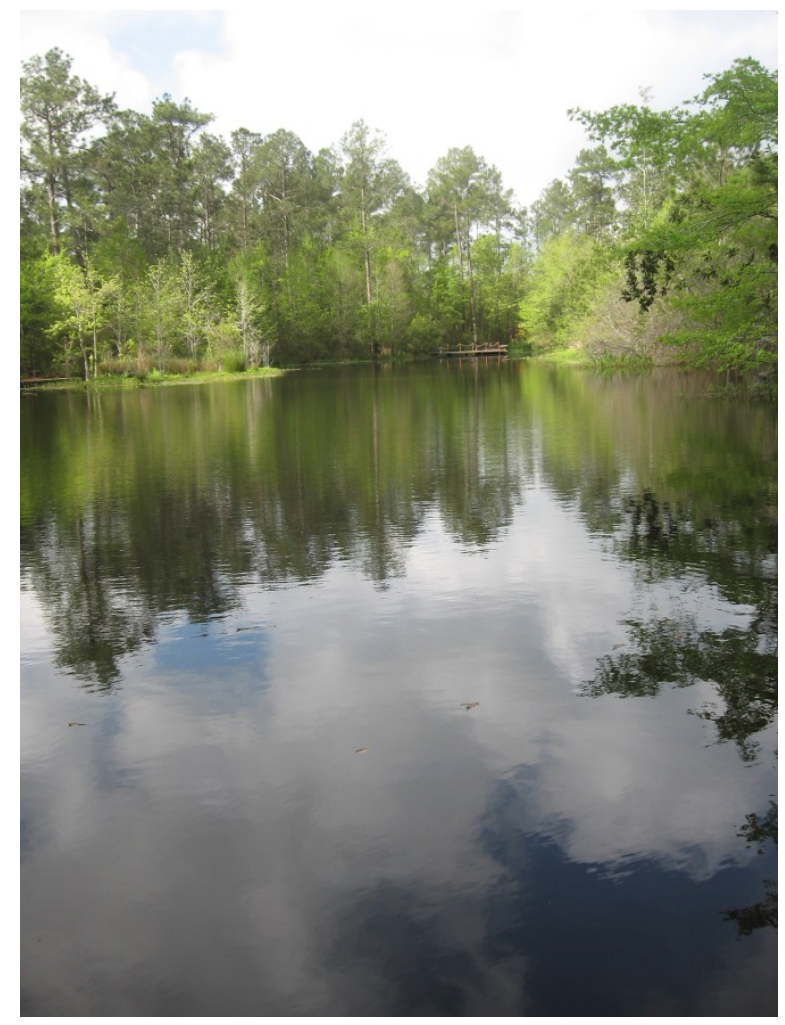

Figure 5. Beaver Pond Exhibit area (Photo :Banu Ozturk Kurtaslan, 2011).

In its early years, The Crosby Arboretum conducted biological surveys for all of the plants that existed on site. In order to do this, Arboretum managers had the property surveyed and established permanent reference markers across the site. These reference markers would framework the entire site into a grid of one hundred foot square plots that could then be 
individually studied. Brass stakes were tamped into the ground at each corner of each grid section. At the top of each stake, a brass plate was stamped with the unique identifiers for that location. In addition to becoming permanent reference markers for subsequent floristic surveys, the brass markers became key alignment points for the locations of structures at the arboretum's Master Plan [12].

On the Table 1, the list of the rich plant species can be seen.

\begin{tabular}{|c|c|c|c|c|c|}
\hline Scientific Name & Common Name & Duration & Habit & Sun & Water \\
\hline Acalypha gracilen & Slender threeseed mercury & Annual & Herb & & \\
\hline Acer barbatum & $\begin{array}{l}\text { Southern sugar maple, Florida maple, Caddo } \\
\text { maple }\end{array}$ & Perennial & Tree & Part-shade & Dry \\
\hline Acer rubrum & Red maple, Scarlet maple & Perennial & Tree & Sun, Part-shade & Moist \\
\hline $\begin{array}{l}\text { Acer rubrum var. } \\
\text { drummondii }\end{array}$ & $\begin{array}{l}\text { Drummond's maple, Drummond red maple, } \\
\text { Swamp maple }\end{array}$ & Perennial & Tree & Part shade & Wet, moist \\
\hline Aesculus pavia & $\begin{array}{l}\text { Scarlet Buckeye, Red buckeye, Firecracker } \\
\text { plant }\end{array}$ & Perennial & Shrub,Tree & Part shade & Wet, moist \\
\hline Agalinis fasciculata & Beach false foxglove & Annual & Herb & & \\
\hline Agalinis purpurea & $\begin{array}{l}\text { Purple false foxglove, Purple gerardia, } \\
\text { Gerardia }\end{array}$ & Annual & Herb & $\begin{array}{l}\text { Sun, Shade, } \\
\text { Part-shade }\end{array}$ & Wet, Moist \\
\hline Agalinis tenuifolia & Slenderleaf false foxglove & Annual & Herb & & \\
\hline Aletris aurea & $\begin{array}{l}\text { Golden colicroot, Colicroot, Star grass, Yellow } \\
\text { colic root }\end{array}$ & Perennial & Herb & & \\
\hline Aletris farinosa & White colicroot, Colic root, Unicorn root & Perennial & Herb & Sun & Moist, Dry \\
\hline Alnus serrulata & $\begin{array}{l}\text { Hazel alder, Brookside alder, Tag alder, } \\
\text { Common alder } \\
\text { Common serviceberry, Downy serviceberry, }\end{array}$ & Perennial & Shrub & $\begin{array}{l}\text { Sun, Shade, } \\
\text { Part-shade } \\
\text { Sun, Shade, }\end{array}$ & Wet, Moist \\
\hline Amelanchier arborea & $\begin{array}{l}\text { Shadbush, Juneberry } \\
\text { Indigo bush, False indigo bush, False indigo, }\end{array}$ & Perennial & Shrub & Part-shade & Dry \\
\hline Amorpha fruticosa & Desert false indigo & Perennial & $\begin{array}{l}\text { Shrub } \\
\text { Grass/Grass- }\end{array}$ & Sun, Part-shade & Moist \\
\hline Andropogon virginicus & $\begin{array}{l}\text { Broomsedge bluestem, Broom-sedge } \\
\text { Devil's walkingstick, Devil's walking-stick, }\end{array}$ & Perennial & like & Part-shade & Moist \\
\hline Aralia spinosa & Prickly Ash, Hercules & Perennial & $\begin{array}{l}\text { Tree } \\
\text { Grass/Grass- }\end{array}$ & Part-shade & Moist \\
\hline Arundinaria gigantea & $\begin{array}{l}\text { Giant cane } \\
\text { Fewflower milkweed, Few-flower milkweed, }\end{array}$ & Perennial & like & Part-shade & Wet \\
\hline Asclepias lanceolata & Red milkweed & Perennial & Herb & Sun & Wet \\
\hline Asclepias longifolia & $\begin{array}{l}\text { Longleaf milkweed } \\
\text { Groundseltree, Sea-myrtle, } \\
\text { Consumptionweed, Eastern baccharis, } \\
\text { Groundsel, Groundsel bush, Salt marsh-elder, }\end{array}$ & Perennial & Herb & & \\
\hline Baccharis halimifolia & Salt bush, Florida groundsel bush & Perennial & Shrub & Part-shade & Wet \\
\hline \multicolumn{6}{|l|}{ Baccharis halimifolia } \\
\hline Balduina uniflora & Oneflower honeycombhead & Perennial & Herb & & \\
\hline Bartonia paniculata & Twining screwstem & Annual & Vine & & \\
\hline Betula nigra & River birch & Perennial & Tree & Part-shade & Moist \\
\hline Bidens aristosa & Bearded beggarticks, Tickseed sunflower & Annual & Herb & & \\
\hline Buchnera americana & American bluehearts, Bluehearts & Annual & Herb & Sun & Moist \\
\hline Callicarpa americana & American beautyberry, French mulberry & Perennial & Shrub & Part-shade & Moist \\
\hline Calopogon pallidus & Pale grasspink & Perennial & Herb & & \\
\hline Calopogon tuberosus & Tuberous grasspink, Grass pink & Perennial & Herb & & \\
\hline Canna flaccida & Bandanna of the Everglades, Golden canna & Perennial & Herb & Sun & Wet \\
\hline
\end{tabular}




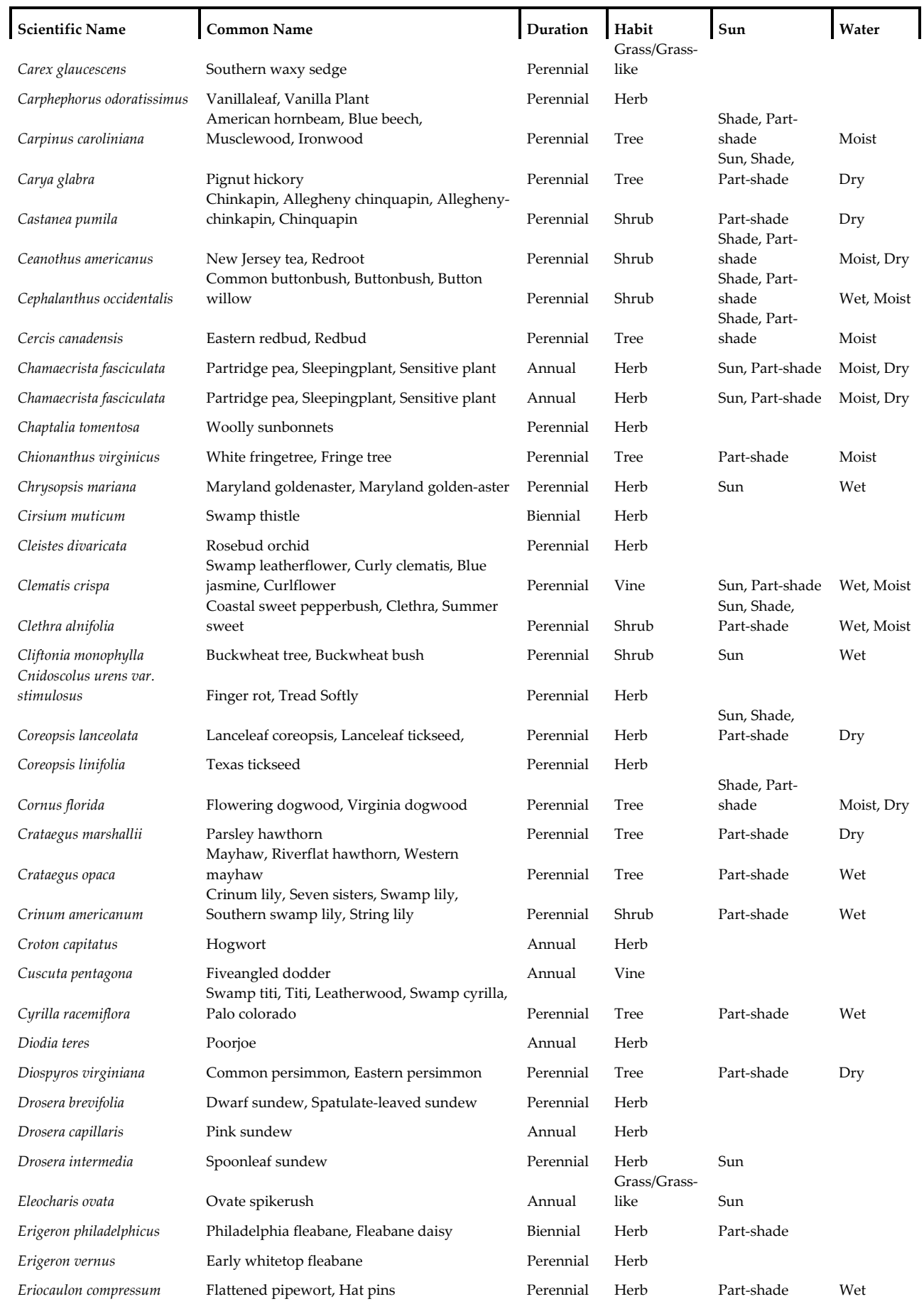




\begin{tabular}{|c|c|c|c|c|c|}
\hline Scientific Name & Common Name & Duration & Habit & Sun & Water \\
\hline Eriocaulon decangulare & Tenangle pipewort, Pipewort & Perennial & Herb & Sun & \\
\hline Erythrina herbacea & $\begin{array}{l}\text { Coralbean, Cherokee bean, Red cardinal } \\
\text { American strawberry-bush, Strawberry bush, } \\
\text { Brook euonymus, Hearts-a-burstin, Bursting- }\end{array}$ & Perennial & Shrub & Sun, Part-shade & Dry \\
\hline Euonymus americanus & heart, Wahoo & Perennial & Shrub & Part-shade & Moist \\
\hline Eupatoriadelphus fistulosus & $\begin{array}{l}\text { Trumpetweed, Queen of the meadow, Hollow } \\
\text { Joe-pye weed, Joe-pye weed }\end{array}$ & Perennial & Herb & Sun & Wet, Moist \\
\hline Eupatorium capillifolium & Dog Fennel, Dogfennel & Perennial & Herb & & \\
\hline Eupatorium perfoliatum & Common boneset & Perennial & Herb & $\begin{array}{l}\text { Sun, Shade, } \\
\text { Part-shade }\end{array}$ & Wet, Moist \\
\hline Eupatorium rotundifolium & Roundleaf thoroughwort & Perennial & Herb & & \\
\hline Fagus grandifolia & American beech & Perennial & Tree & $\begin{array}{l}\text { Shade, Part- } \\
\text { shade }\end{array}$ & Moist \\
\hline Galium tinctorium & Stiff marsh bedstraw & Perennial & Herb & Part-shade & \\
\hline Gaylussacia dumosa & Dwarf huckleberry & Perennial & Shrub & Part-shade & \\
\hline Gelsemium sempervirens & $\begin{array}{l}\text { Carolina jessamine, Yellow jessamine, Evening } \\
\text { trumpetflower, Poor man's rope }\end{array}$ & Perennial & Vine & $\begin{array}{l}\text { Sun, Part-shade } \\
\text { Shade, Part- }\end{array}$ & Moist \\
\hline Geranium maculatum & Spotted geranium, Wild geranium, Cranesbill & Perennial & Herb & shade & Moist \\
\hline Gordonia lasianthus & $\begin{array}{l}\text { Gordonia, Loblolly bay } \\
\text { Two-wing silverbell, Silver bell, Two-winged } \\
\text { silverbell, Snowdrop tree, American }\end{array}$ & Perennial & Shrub & Sun & Moist \\
\hline Halesia diptera & snowdrop tree & Perennial & Shrub, Tree & $\begin{array}{l}\text { Part-shade } \\
\text { Shade, Part- }\end{array}$ & Dry \\
\hline Hamamelis virginiana & Witch hazel, American witch hazel & Perennial & Tree & shade & Moist, Dry \\
\hline Helenium vernale & Savannah sneezeweed & Perennial & Herb & & \\
\hline Helianthus angustifolius & Swamp sunflower & Perennial & Herb & Part-shade & Wet \\
\hline Hibiscus aculeatus & $\begin{array}{l}\text { Comfortroot, Big thicket hibiscus, Pineland } \\
\text { hibiscus } \\
\text { Rose-mallow, Rosemallow, Woolly mallow, }\end{array}$ & Perennial & Herb & Sun, Part-shade & Moist \\
\hline Hibiscus lasiocarpos & Wooly Rose-mallow & Perennial & Shrub & Sun & Wet \\
\hline Hydrangea quercifolia & Oakleaf hydrangea, Oak-leaf hydrangea & Perennial & Shrub & Shade & Moist \\
\hline Hypericum crux-andreae & St. Peterswort & Perennial & Subshrub & Part-shade & \\
\hline Hypericum gentianoides & Orangegrass, Pineweed & Annual & Herb & Part-shade & \\
\hline Hypericum gymnanthum & Claspingleaf St. Johnswort & Perennial & Herb & & \\
\hline Hypericum tetrapetalum & Fourpetal St. Johnswort & Perennial & Subshrub & & \\
\hline Hypoxis hirsuta & $\begin{array}{l}\text { Common goldstar, Eastern yellow star-grass, } \\
\text { Yellow star-grass }\end{array}$ & Perennial & Herb & $\begin{array}{l}\text { Sun, Shade, } \\
\text { Part-shade }\end{array}$ & Dry \\
\hline Ilex amelanchier & Sarvis holly, Swamp holly & Perennial & Shrub & Part-shade & Wet \\
\hline Ilex coriacea & $\begin{array}{l}\text { Large gallberry, Bay-gall bush, Ink-berry holly } \\
\text { Possumhaw, Possumhaw Holly, Deciduous }\end{array}$ & Perennial & Shrub & Part-shade & Moist \\
\hline Ilex decidua & Holly, Winterberry, Deciduous yaupon & Perennial & Shrub & Sun, Part-shade & Moist \\
\hline Ilex glabra & Inkberry, Gallberry & Perennial & Shrub & Part-shade & Wet, Moist \\
\hline Ilex myrtifolia & Myrtle dahoon, Myrtle leaf holly, Myrtle holly & Perennial & Shrub & $\begin{array}{l}\text { Part-shade } \\
\text { Sun, Shade, }\end{array}$ & Moist \\
\hline Ilex opaca & American holly, Christmas holly & Perennial & Tree & Part-shade & Wet, Dry \\
\hline Ilex verticillata & $\begin{array}{l}\text { Common winterberry, Michigan holly, Black } \\
\text { alder }\end{array}$ & Perennial & Tree & $\begin{array}{l}\text { Sun, Shade, } \\
\text { Part-shade } \\
\text { Sun, Shade, }\end{array}$ & $\begin{array}{l}\text { Wet, } \\
\text { Moist, Dry }\end{array}$ \\
\hline Ilex vomitoria & $\begin{array}{l}\text { Yaupon, Yaupon holly, Cassina } \\
\text { Florida anisetree, Florida anise, Anise tree, }\end{array}$ & Perennial & Shrub, Tree & Part-shade & Moist, Dry \\
\hline Illicium floridanum & Stinkbush & Perennial & Shrub & Part-shade & \\
\hline Ipomoea pandurata & $\begin{array}{l}\text { Man of the earth, Wild Potato, Wild sweet } \\
\text { potato }\end{array}$ & Perennial & Herb & $\begin{array}{l}\text { Sun, Shade, } \\
\text { Part-shade }\end{array}$ & Moist \\
\hline
\end{tabular}




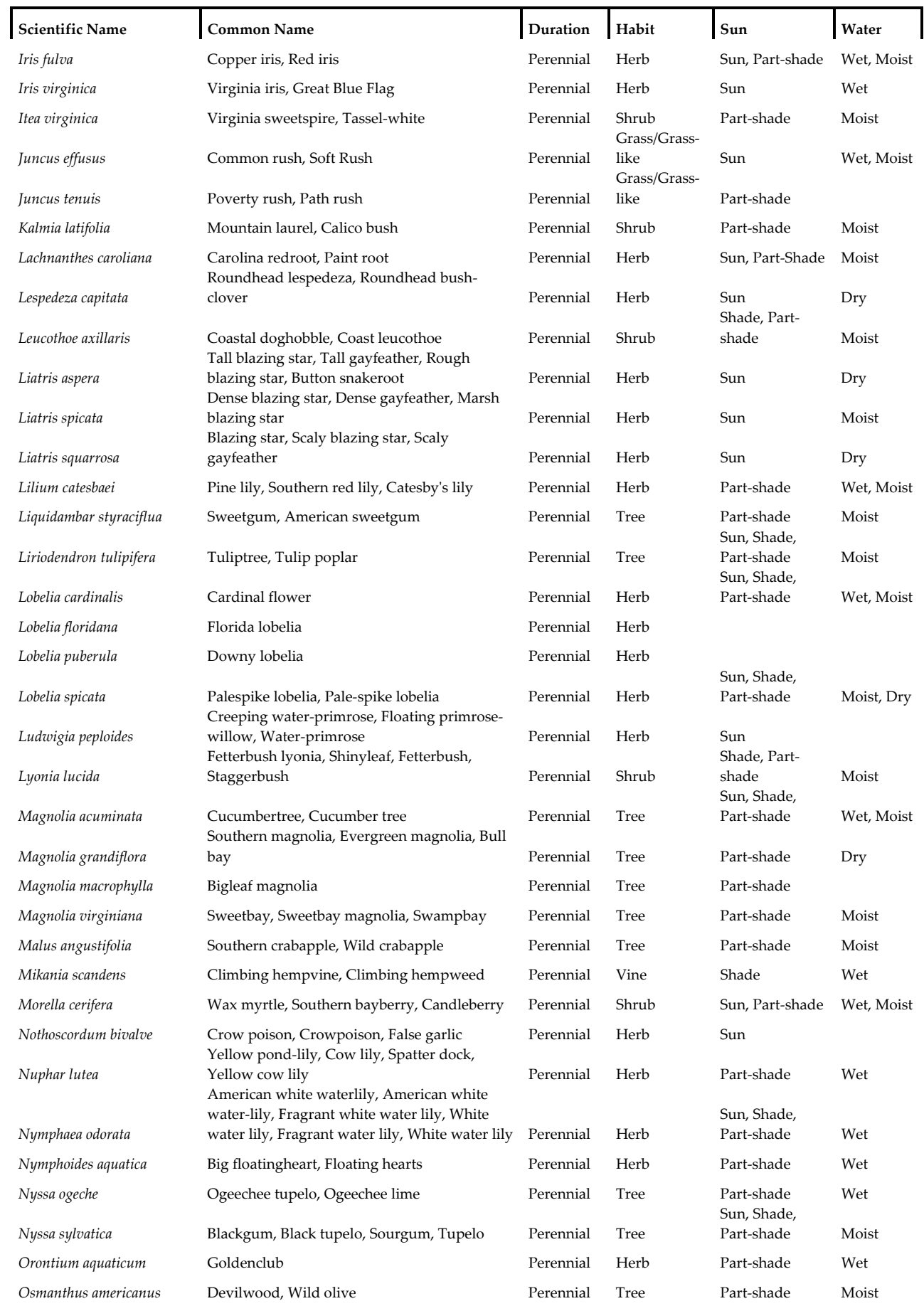




\begin{tabular}{|c|c|c|c|c|c|}
\hline Scientific Name & Common Name & Duration & Habit & Sun & Water \\
\hline Osmunda cinnamomea & Cinnamon fern & Perennial & Herb, Fern & $\begin{array}{l}\text { Sun, Shade, } \\
\text { Part-shade }\end{array}$ & Wet, Moist \\
\hline Oxalis stricta & Common yellow oxalis, Yellow Wood-sorrel & Perennial & Herb & Sun & Dry \\
\hline Oxypolis filiformis & Water cowbane & Perennial & Herb & & \\
\hline Panicum virgatum & Switchgrass, Wand panic grass & Perennial & $\begin{array}{l}\text { Grass/Grass- } \\
\text { like } \\
\text { Grass/Grass- }\end{array}$ & Sun, Part-shade & Moist, Dry \\
\hline Paspalum plicatulum & Brownseed paspale, Brownseed paspalum & Perennial & like & Part-shade & Moist \\
\hline Peltandra virginica & $\begin{array}{l}\text { Green arrow arum, Tuckahoe, Arrow arum, } \\
\text { Green arrow-arum }\end{array}$ & Perennial & Herb & Part-shade & Wet \\
\hline Persea borbonia & Redbay, Red bay & Perennial & Tree & Part-shade & Moist \\
\hline Persea palustris & Swamp bay & Perennial & Shrub, Tree & & \\
\hline Phlox pilosa & Downy phlox, Prairie phlox, Fragrant phlox & Perennial & Herb & Sun, Part-shade & Dry \\
\hline Photinia pyrifolia & Red chokeberry, Red chokecherry & Perennial & Shrub & Sun & Moist \\
\hline Physostegia virginiana & $\begin{array}{l}\text { Fall obedient plant, Obedient plant, False } \\
\text { dragonhead }\end{array}$ & Perennial & Herb & $\begin{array}{l}\text { Sun, Shade, } \\
\text { Part-shade }\end{array}$ & Moist \\
\hline Pinguicula lutea & Yellow butterwort & Perennial & Herb & Sun & Moist \\
\hline Pinus elliottii & Slash pine & Perennial & Tree & Part-shade & Moist \\
\hline Pinus glabra & Spruce pine & Perennial & Tree & Part-shade & Moist \\
\hline Pinus palustris & Longleaf pine, Georgia pine & Perennial & Tree & Sun & Dry \\
\hline Pinus taeda & $\begin{array}{l}\text { Loblolly pine, Old field pine, Bull pine, } \\
\text { Rosemary pine }\end{array}$ & Perennial & Tree & Part-shade & Dry \\
\hline Pluchea foetida & Stinking camphorweed & Perennial & Herb & & \\
\hline Pogonia ophioglossoides & $\begin{array}{l}\text { Beard flower, Rose pogonia orchid, Snake- } \\
\text { mouth orchid, Snakemouth Moist }\end{array}$ & Perennial & Herb & Sun & Wet, \\
\hline Polygala cruciata & Candy root, Drumheads & Annual & Herb & & \\
\hline Polygala incarnata & Procession flower & Annual & Herb & Sun & Dry \\
\hline Polygala lutea & Candy Weed, Orange milkwort & Biennial & Herb & & \\
\hline Polygala mariana & Maryland milkwort & Annual & Herb & & \\
\hline Polygala nana & Candyroot & Annual & Herb & & \\
\hline Polygala ramosa & $\begin{array}{l}\text { Low pinebarren milkwort, Yellow savannah } \\
\text { milkwort }\end{array}$ & $\begin{array}{l}\text { Annual } \\
\text { Annual, }\end{array}$ & Herb & & \\
\hline Polypremum procumbens & Juniper leaf & Perennial & Herb & & \\
\hline Pontederia cordata & Pickerelweed, Pickerel Weed & Perennial & Herb & Sun, Part-shade & Wet, Moist \\
\hline Prunus angustifolia & Chickasaw plum, Sandhill plum & Perennial & Tree & $\begin{array}{l}\text { Sun, Part-shade } \\
\text { Sun, Shade, }\end{array}$ & Dry \\
\hline Prunus serotina & Black cherry, Rum cherry & Perennial & Tree & Part-shade & Moist, Dry \\
\hline Pteridium aquilinum & $\begin{array}{l}\text { Western bracken fern, Bracken fern, Western } \\
\text { bracken, Bracken }\end{array}$ & Perennial & Herb, Fern & $\begin{array}{l}\text { Shade, Part- } \\
\text { shade }\end{array}$ & $\begin{array}{l}\text { Wet, } \\
\text { Moist, Dry }\end{array}$ \\
\hline Ptilimnium capillaceum & Herbwilliam & Annual & Herb & & \\
\hline Pyrrhopappus carolinianus & Carolina desert-chicory & Annual & Herb & & \\
\hline Quercus alba & $\begin{array}{l}\text { White oak, Northern white oak, Stave Oak, } \\
\text { Ridge White Oak, Forked-leaf White Oak }\end{array}$ & Perennial & Tree & $\begin{array}{l}\text { Sun, Shade, } \\
\text { Part-shade }\end{array}$ & Moist, Dry \\
\hline Quercus falcata & $\begin{array}{l}\text { Southern red oak, Spanish oak } \\
\text { Bluejack oak, Sandjack oak, Upland willow }\end{array}$ & Perennial & Tree & Part-shade & Dry \\
\hline Quercus incana & $\begin{array}{l}\text { oak, Cinnamon oak, Shin oak, Turkey oak } \\
\text { Laurel oak, Swamp laurel oak, Darlington oak, } \\
\text { diamond-leaf oak, laurel-leaf oak, water oak, }\end{array}$ & Perennial & Shrub, Tree & Part-shade & Dry \\
\hline Quercus laurifolia & obtuse oak & Perennial & Tree & Part-shade & \\
\hline Quercus marilandica & Blackjack oak, Barren oak, black oak, jack oak & Perennial & Tree & Part-shade & Dry \\
\hline Quercus michauxii & Swamp chestnut oak, Basket oak, Cow oak & Perennial & Tree & Part-shade & Moist \\
\hline
\end{tabular}




\begin{tabular}{|c|c|c|c|c|c|}
\hline Scientific Name & Common Name & Duration & Habit & Sun & Water \\
\hline Quercus nigra & Water oak & Perennial & Tree & Part-shade & Wet \\
\hline Quercus pagoda & Cherrybark oak & Perennial & Tree & & \\
\hline Quercus phellos & Willow oak & Perennial & Tree & Part-shade & Moist \\
\hline Quercus virginiana & Coastal live oak, Southern live oak, Live oak & Perennial & Tree & Sun, Part-shade & Moist \\
\hline Rhapidophyllum hystrix & Needle palm, Blue palmetto & Perennial & Shrub & Part-shade & \\
\hline Rhexia alifanus & Savannah meadowbeauty & Perennial & Herb & & \\
\hline Rhexia lutea & $\begin{array}{l}\text { Yellow meadow beauty, Yellow } \\
\text { meadowbeauty }\end{array}$ & Perennial & Herb & & \\
\hline Rhexia mariana & $\begin{array}{l}\text { Maryland meadowbeauty, Maryland } \\
\text { meadow-beauty, Meadow beauty }\end{array}$ & Perennial & Herb & Part-shade & Moist \\
\hline Rhexia petiolata & Fringed meadowbeauty & Perennial & Herb & & \\
\hline Rhexia virginica & Meadow beauty, Handsome Harry & Perennial & Herb & Part-shade & Wet \\
\hline Rhododendron austrinum & $\begin{array}{l}\text { Orange azalea, Florida azalea, Yellow azalea } \\
\text { Mountain azalea, Wild azalea, Honeysuckle } \\
\text { azalea, Piedmont azalea, Sweet azalea, Hoary }\end{array}$ & Perennial & Shrub & Part-shade & Dry \\
\hline Rhododendron canescens & $\begin{array}{l}\text { azalea, Southern pinxterflower } \\
\text { Winged sumac, Shining sumac, Flameleaf }\end{array}$ & Perennial & Shrub & Part-shade & Dry \\
\hline Rhus copallinum & $\begin{array}{l}\text { sumac } \\
\text { Starrush whitetop, Star sedge, White-topped }\end{array}$ & Perennial & $\begin{array}{l}\text { Shrub } \\
\text { Grass/Grass- }\end{array}$ & Sun & Dry \\
\hline Rhynchospora colorata & $\begin{array}{l}\text { sedge, Whitetop sedge } \\
\text { Shortbristle horned beaksedge, Horned }\end{array}$ & Perennial & $\begin{array}{l}\text { like } \\
\text { Grass/Grass- }\end{array}$ & Sun, Part-shade & Wet \\
\hline Rhynchospora corniculata & Beakrush & Perennial & $\begin{array}{l}\text { like } \\
\text { Grass/Grass- }\end{array}$ & Sun & \\
\hline Rhynchospora glomerata & Clustered beaksedge, Cluster Beak-rush & Perennial & like & & \\
\hline Rubus argutus & Sawtooth blackberry & Perennial & Shrub & & \\
\hline Rubus trivialis & $\begin{array}{l}\text { Dewberry, Southern dewberry } \\
\text { Black-eyed Susan, Common black-eyed Susan, }\end{array}$ & Perennial & Herb & $\begin{array}{l}\text { Sun, Part-shade } \\
\text { Sun, Shade, }\end{array}$ & Moist, Dry \\
\hline Rudbeckia hirta & Brown-eyed Susan & Annual & Herb & $\begin{array}{l}\text { Part-shade } \\
\text { Sun, Shade, }\end{array}$ & Moist, Dry \\
\hline Sabal minor & Dwarf palmetto, Palmetto, Bush palmetto & Perennial & Shrub & Part-shade & Moist, Dry \\
\hline Sabatia brachiata & $\begin{array}{l}\text { Narrowleaf rose gentian } \\
\text { Texas star, Rose gentian, Meadow pink, }\end{array}$ & Biennial & Herb & & \\
\hline Sabatia campestris & Prairie rose-gentian, Prairie sabatia & Annual & Herb & Part-shade & Dry \\
\hline Sagittaria graminea & Grassy arrowhead, Grass-leaf arrowhead & Perennial & Herb & & \\
\hline Salix nigra & $\begin{array}{l}\text { Black willow, Gulf black willow } \\
\text { Pitcher sage, Big blue sage, Azure sage, Giant }\end{array}$ & Perennial & Tree & $\begin{array}{l}\text { Sun, Shade, } \\
\text { Part-shade }\end{array}$ & Wet, Moist \\
\hline Salvia azurea & blue sage, Blue sage & Perennial & Herb & $\begin{array}{l}\text { Part-shade } \\
\text { Sun, } \\
\text { Shade,Part- }\end{array}$ & Dry \\
\hline Salvia lyrata & $\begin{array}{l}\text { Lyreleaf sage, Cancer weed } \\
\text { Flycatcher, Yellow pitcher plant, Yellow }\end{array}$ & Perennial & Herb & shade & Moist,Dry \\
\hline Sarracenia alata & trumpet, Yellow trumpets & Perennial & Herb & Wet & \\
\hline Sarracenia psittacina & Parrot pitcherplant & Perennial & Subshrub & & \\
\hline Sassafras albidum & Sassafras & Perennial & Tree & $\begin{array}{l}\text { Sun, Shade, } \\
\text { Part-shade } \\
\text { Shade, Part- }\end{array}$ & Moist \\
\hline Saururus cernuus & $\begin{array}{l}\text { Lizard's tail, Lizard's-tail, Breast weed } \\
\text { Woolgrass, Cottongrass bulrush, Marsh }\end{array}$ & Perennial & $\begin{array}{l}\text { Herb } \\
\text { Grass/Grass- }\end{array}$ & shade & Wet, Moist \\
\hline Scirpus cyperinus & $\begin{array}{l}\text { bulrush, Teddybear paws } \\
\text { Helmet-flower, Rough Skullcap, Common }\end{array}$ & Perennial & like & Sun & Wet \\
\hline Scutellaria integrifolia & Large Skullcap, Tall Skullcap & Perennial & Herb & Sun & Wet \\
\hline Serenoa repens & $\begin{array}{l}\text { Saw palmetto } \\
\text { Narrowleaf blue-eyed grass, Narrow-leaf }\end{array}$ & Perennial & Shrub & Part-shade & \\
\hline Sisyrinchium angustifolium & blue-eyed-grass, Bermuda blue-eyed grass, & Perennial & Herb & Sun, Part-shade & Wet, Moist \\
\hline
\end{tabular}




\begin{tabular}{|c|c|c|c|c|c|}
\hline Scientific Name & $\begin{array}{l}\text { Common Name } \\
\text { Blue-eyed grass }\end{array}$ & Duration & Habit & Sun & Water \\
\hline Smilax laurifolia & Laurel greenbriar, Laurel greenbrier & Perennial & Vine & Part-shade & Wet \\
\hline Smilax pumila & $\begin{array}{l}\text { smilax, Dwarf greenbrier } \\
\text { Lanceleaf greenbrier, Southern smilax, }\end{array}$ & Perennial & Vine & Part-shade & Dry \\
\hline Smilax smallii & Jacksonvine, Jacksonbrier & Perennial & Vine & Sun, Part-shade & Moist \\
\hline Solidago altissima & $\begin{array}{l}\text { Tall Goldenrod, Late goldenrod, Canadian } \\
\text { goldenrod, Canada goldenrod }\end{array}$ & Perennial & Herb & $\begin{array}{l}\text { Shade, Part- } \\
\text { shade }\end{array}$ & Moist \\
\hline Solidago gigantea & goldenrod, Late goldenrod, Early goldenrod & Perennial & Herb & Part-shade & \\
\hline Solidago odora var. odora & Anisescented goldenrod & Perennial & Herb & & \\
\hline Solidago patula & $\begin{array}{l}\text { Roundleaf goldenrod } \\
\text { Wrinkleleaf goldenrod, Wrinkle-leaf } \\
\text { goldenrod, Rough-leaved goldenrod, } \\
\text { Roughleaf goldenrod, } \\
\text { Rough-stemmed goldenrod, Roughstem }\end{array}$ & Perennial & Herb & $\begin{array}{l}\text { Sun, Shade, } \\
\text { Part-shade }\end{array}$ & Wet, Moist \\
\hline Solidago rugosa & $\begin{array}{l}\text { goldenrod } \\
\text { Nodding lady's tresses, Ladies' tresses, }\end{array}$ & Perennial & Herb & Sun & Wet \\
\hline Spiranthes cernua & Nodding ladies' tresses orchid & Perennial & Herb & Sun & Moist \\
\hline Spiranthes praecox & Greenvein lady's tresses & Perennial & Herb & & \\
\hline Stokesia laevis & Stokes aster & Perennial & Herb & Sun, Part-shade & Moist \\
\hline Stylosanthes biflora & $\begin{array}{l}\text { Sidebeak pencilflower } \\
\text { American snowbell, American snowbells, }\end{array}$ & Perennial & Herb & & \\
\hline Styrax americanus & $\begin{array}{l}\text { American silverbells, Big-leaf snowbell, Storax } \\
\text { Horsesugar, Common sweetleaf, Sweetleaf, }\end{array}$ & Perennial & Tree & Part-shade & Wet \\
\hline Symplocos tinctoria & Yellowwood & Perennial & Tree & Part-shade & Wet \\
\hline Taxodium ascendens & Pond cypress & Perennial & Tree & Sun & Moist \\
\hline Taxodium distichum & $\begin{array}{l}\text { Bald cypress, Baldcypress, Common bald } \\
\text { cypress, Southern bald cypress }\end{array}$ & Perennial & Tree & $\begin{array}{l}\text { Sun, Part-shade } \\
\text { Shade, Part- }\end{array}$ & Moist \\
\hline Toxicodendron radicans & Eastern poison ivy, Poison ivy, Poison oak & Perennial & Shrub, Vine & shade & Moist \\
\hline Ulmus alata & Winged elm & Perennial & Tree & Part-shade & Dry \\
\hline Vaccinium arboreum & Farkleberry, Tree sparkleberry, Sparkleberry & Perennial & Tree & Part-shade & Dry \\
\hline Vaccinium darrowii & Darrow's blueberry, Evergreen blueberry & Perennial & Shrub & Part-shade & Moist \\
\hline Vaccinium elliottii & Elliott's blueberry & Perennial & Shrub & & \\
\hline Viburnum nudum & Possumhaw viburnum, Possumhaw & Perennial & Shrub & $\begin{array}{l}\text { Sun, Shade, } \\
\text { Part-shade }\end{array}$ & Wet, Moist \\
\hline Viola septemloba & Southern coastal violet & Perennial & Herb & & \\
\hline Vitis rotundifolia & Muscadine, Scuppernong grape & Perennial & Vine & Part-shade & Moist \\
\hline $\begin{array}{l}\text { Wisteria frutescens } \\
\text { Woodwardia areolata }\end{array}$ & $\begin{array}{l}\text { American wisteria, Texas wisteria, Kentucky } \\
\text { wisteria } \\
\text { Netted chainfern, Chain fern, Netted chain } \\
\text { fern }\end{array}$ & Perennial & Herb, Fern & $\begin{array}{l}\text { Sun, Shade, } \\
\text { Part-shade } \\
\text { Shade, Part- } \\
\text { shade }\end{array}$ & $\begin{array}{l}\text { Moist } \\
\text { Wet, Moist }\end{array}$ \\
\hline Woodwardia virginica & $\begin{array}{l}\text { Virginia chain fern, Virginia chainfern } \\
\text { Coastal plain yelloweyed grass, Coastalplain }\end{array}$ & Perennial & Herb, Fern & Part-shade & Wet, Moist \\
\hline Xyris ambigua & yelloweyed grass & Perennial & Herb & & \\
\hline Xyris difformis & Bog yelloweyed grass & Perennial & Herb & & \\
\hline Zigadenus glaberrimus & Camas, Sandbog deathcamas & Perennial & Herb & & \\
\hline
\end{tabular}

Table 1. The list of the plant species at the Crosby Arboretum (http://www.wildflower.org/collections/printable.php?collection=Organization_817) 
The wildlife patterns at the arboretum as below:

\section{Animals:}

Rabbit

Beaver

Muscrat

Opposum (sign)

Raccoon (sign)

Skunk (sign)

Fox (sign)

Coyote (reported from neighbours)

Feral (\& pet) dogs \& cats

Squirrel

Rat (spp. unknown)

Mice (spp. unknown)

Shrew (spp. unknown)

Rat snake (spp. unknown)

Diamond-Backed water snake

Copperhead Snake

Water Moccasin Snake

Garter Snake

Box Turtle

\section{Birds:}

Waterfowl

Bobwhite Quail

Turkey

Woodcock

Great Blue Heron

Little Blue Heron

Kingfisher

Red-tailed Hawk

English Sparrow

Starling

Red-Headed Woodpecker

Mourning Dove

Bobwhite quail

Mockingbird

Robin

Brown Thrasher

\section{Other:}

Crayfish (probably more than one species)

Insects ad infinitum 


\section{The esthetic significance of the arboretum}

Gardener William Robinson (1838-1935) was one of the first to abstract nature in garden designs. Within natural environments, Robinson was very sensitive to landscape design characteristics, including vegetation colors, forms, and textures. Robinson advocated studying the spatial composition of a natural landscape in his book The Wild Garden by observing how boundaries of a space were contained by the trees and shrubs. Similarly, Winston Spirn (1984) and further stipulates that landscape architects play a role in creating "conspicuous expression and visible interpretation" of landscape, and that the use of artistic interpretation plays an important role. Spirn states that "the current understanding of nature and culture as comprising interwoven processes that exhibit a complex, underlying order which holds across vast scales of space and time, not only demands a new aesthetic, new forms, and new modes of design, construction, and cultivation, but also prompts a fresh appreciation for the forms of the past and the processes by which they were created." Calling for a new design aesthetic meant its acceptance and embracement by the general public was needed as well.

In recent years, considerable debate within the design profession about aesthetics and values of ecological design, particularly for urban systems. On one hand, landscape architects are challenged to develop more ecologically sensitive projects, while one the other hand, they are drawing on the richly established tradition of visually aesthetic design. According to Mozingo (1997) [20] ecological designers must consider cultural needs and to incorporate current perceptions of aesthetics and beauty.

As it is mentioned above, there are some water exhibits in the Crosby Arboretum. For ecosystems that feature riparian or wetland systems, water is an important element in ecological design interpretation. It's surface, color, form, reflectiveness or movement are landscape elements in terms of bio-physical perspectives. With its aesthetic quality and the image and symbol that it offers [12]. It creates a sense of a place. I is often used to symbolize things in iterature. Water is a universal symbol of change and is often present at turning points in a story. Since water is often sign of life many times water represents life. Fresh water can represent good health and bad water symbolizes bad health. Water can also mean "purity and cleansing" (http:// symbolis.wikia.com/wiki/Water). For developing the relationships of people with water, every form of land use must be based upon a clear understanding of the relationships of the water within the physical characteristics unique to each place [23].

Art elements and principles can be found near natural small streams. While there is some degree of presence of all of design principles (balance, proportion, rhythm, emphasis, and unity) and elements (point, line, form, shape and space, movement, color, pattern, and texture) found within areas of small streams, the artistic elements such as line and rhythm dominate this landscape type more strongly than others. "Line" is one of the more apparent design elements of a channelized water system. Most small streams have a clearly defined bank edge where the water travels. Stream lines which are horizontal features, contrast strongly vertical lines of adjacent mature trees. These line forms create a different emotional 
experience for the viewer, as straight streams provide a distant vanishing point and meandering streams create a sense of mystery as to what is around the next bend. Like this, many natural features such as tree trunks in a dense forest create a sense of "rhythm," as a design principal. In a forested system, a creek becomes a contrast as it is a different material from the surrounding vegetation, rocks or soil. But it also serves importantly as a rhythmic item which literally flows from one part of the landscape scene to the next. The "size" of a landscape element in proportion to its surrounding features is an important principle of design. Small creek systems not only need to serve their hydrological role, but also serve their aesthetic parameters. The design principles such as line, rhythm, and scale are permanent features for small creeks and streams but many of these can be temporal in nature. However, in the fall season "colors" can change to brightly colored reds and oranges for deciduous trees and shrubs. Seasonal changes affect the qualities of other design criteria, especially spatial formations and scale. When leaves fall in the winter months the spatial dynamics of the landscapes change from dense layers of shrub and ground vegetation to an open landscape type. The surrounding land is more easily viewed and the ground plane is much more apparent. "Scale" also changes in winter months, and a small stream channel becomes even smaller in the greater landscape [12].

Mozingo (1997) [20] writes that "examining ecological design in tandem with landscapes of notable aesthetic quality elucidates the difficulties in reconciling their conception of visibility, temporality, reiterated form, expression and metaphor." She neatly summarizes their premises into these 5 clearly defined principles. She stipulates that ecological landscapes have inherent social acceptance problems on their own accord and must become an accepted icon or symbol by the general public to become broadly accepted. In the study of Brzuszek and Clark (2009) [10], for the Crosby Arboretum visitors, a questionnaire was designed by deriving from Mozingo's principles. It aimed to examine some qualities of Crosby arboretum such as visibility, care, orderliness, human presence, meaning were analyzed for the correlation between their value and perception for each. According to the results, visitors;

1. Believe that a landscape should blend into its surrounding environment and see this at Crosby;

2. Believe that a landscape should appear cared for and find Crosby to be well cared for;

3. Believe that landscapes need not be necessarily orderly in their appearance and find Crosby to be somewhat too orderly;

4. Are somewhat neutral on the question of having human forms of expression in the landscape but detected a modest presence at Crosby; and

5. Place high value on the need for meaning in the landscape and give Crosby high marks.

Crosby Arboretum's project designers merged a symbiotic interplay between the vegetation patterns and physical processes of the arboretum site with the patterns and processes found in local plant communities. Selecting its local watershed as its genius loci, the landscape exhibits of Pinecote (the public interpretive center of the arboretum) are designed to be "compressed, dramatic expressions of the natural features common to the Piney Woods of the Deep South" [11]. To accomplish this, project designers, Andropogon Associates Ltd. 
and Edward L. Blake Jr., merged a symbiotic interplay between the site's vegetation patterns and physical processes with natural patterns found in local plant communities [10].

While analyzing the aesthetic qualities of the Crosby Arboretum, it is useful to take a look at the design principles of Pinecote Pavilion: Pinecote Pavilion is recognized by the Mississippi Department of Archives and History as a Mississippi Landmark (http://www.crosbyarboretum.msstate.edu/ pages/pinecote.php). Euine Fay Jones (19212004), the architect of Pinecote Pavilion described the architectural qualities and design principles of the pavilion by this means: "Architecturally, the Pavilion is a symmetrical shed, resting on a base of earth-toned brick, surrounded by earth, water, and trees. The brick pattern expresses the basic building module-the composition and arrangement of all the vertical columns. The all-wood structure is built of indigenous material, native pine, and is fastened together with nails, dowels, and metal connections. There is complete exposure of every construction element, all visible from within and without. Every framing member, every beam, brace, and connection is absolutely necessary to achieve structural stability. The building is ordered by a geometric theme-a step-edged pattern that defines the outline of the base and the roof's outer edges. Many smaller elements, for lighting and display, are shaped and detailed to reflect and reinforce the characteristic geometry - to build a strong relationship of each part to the whole and to achieve organic unity. As the vertical supports rise from the brick pavement, there is a spreading-out of structural members and a progressively thinning-out of roof decking toward the edges of the hovering roof. There is a transition in the sheltering overhead arrangement, accented by a central skylight, from close and dense to open and fragile. This is analogous to the organic unfolding or blossoming of so many forms of botanical growth. The imbricated pattern of wood shingles also emulate and recall many of natures' surfaces - the bark of trees and the wings of birds. All wood is stained and the metal painted in colors that harmonize with the earth and plants. Nothing has been added to the structure as mere decoration. Ornamentation or decorative enrichment will come from the ever-changing patterns of light and shadows that play on the closely-spaced structural elements as the sun and moon move across the sky. Time of day and seasonal changes will modify the shadows that frame the light and will keep the spaces in and around the Pavilion vital and alive, continuously enhancing the poetics of revealed construction."

E. Fay Jones, FAIA, of Fayetteville, Arkansas, designed all of Pinecote's original buildings. His modest but stunning Pinecote Pavilion won an Honor Award from the American Institute of Architects (AIA) in 1990. That same year, the AIA recognized Fay Jones with its highest individual honor, the Gold Medal. (http://www.crosbyarboretum.msstate.edu/ pages/visitors.php)

\section{The recreational significance of the arboretum}

Green areas stand out with their qualities of meeting the recreational needs of the people, both in the city and its vicinity. Especially, arboretums serve various recreational opportunities which are related to education and nature conservation. When these activities 
are combined with the other attractive activities and events which are for every age groups succesful results.

The variety and richness of flora and fauna living together in different habitats at arboretums provides dynamic tools and resources creating great opportunities for crosscurricular learning. Knowing an outdoor site and identifying the resources available are important to insure the success of this type of program. Nature teaches many lessons about diversity and learning to live together and science helps people investigate questions [24]. The Crosby Arboretum is dedicated to educating the public about their environment as well. Pinecote, serves as the arboretum's public interpretive center. It provide environmental and botanical research opportunities and offer cultural, scientific and recreational programs. The Crosby Arboretum offers a continual schedule of programs and events designed to educate the public about their environment, and to celebrate nature. A festival called "Piney Woods Heritage Festival" is organized at the arboretum each November. Quarterly native plant sales are held. The Arboretum also offers guided and self-guided tours. So that people can observe the threatened or endangered species of plants and wildlife are present at throughout the Arboretum's preserves.

Arboretum website, entry signage and interpretive trail information prepares visitors to the exhibition of native plants in respective plant communities, by setting this tone, visitors understand the context of the facility and better appreciate the educational mission [10]. So, ecologically designed facilities conveys their purpose in promotional literature and interpretive signage this way, the inherent value of those landscapes is conveyed to the visitors. This is an important point for ecologically designed facilities [12].

Central to the arboretum's educational mission was the decision to display native plants in representative plant communities suitable to the site. The Schematic Master Plan outlined the some design principles and almost all the principles serve the educational mission.

At the arboretum, trail systems throughout the site allow visitors to experience more than 50 naturally occurring and human-created regional plant habitats. As the Master Plan stipulates, "Pinecote, a place where the land expresses itself, is the exhibit. Its Master Plan is the organizing framework for the needs and actions off all who use its landscape. This interplay between man and the land organizes the thematic composition of Pinecote's landscape exhibits" [21] [10]. On the walking journey at The Crosby Arboretum the visitor could experience and learn about Gulf Coast landscape. They can take a stroll around the Pond Journey to discover the diversity of life in wetland habitats, visit the South Savanna Exhibit to see carnivorous pitcher plants, and enjoy the wonder of the Pinecote Pavilion, a Mississippi Landmark. Interpretative signs along the trails describe the flora, fauna and cultural history of the Piney Woods region of Mississippi (Figure 6).

At Crosby, the Pavilion is a gathering place. This simple, open building marks a place to be used for many activities. It is a starting point for nature walks, for talks and discussions about important things in the environment and natural world, a place for exhibits and artistic performance, and a setting for social gatherings. (http://www.crosbyarboretum. msstate.edu/pages/pinecote.php). However, a new education building, designed by 
Mississippi architect Tom Howorth, at the arboretum is being built for educational activities (Figure 7).

\section{Some of the recreational events at Crosby Arboretum (2012):}

\section{Special events:}

- $\quad$ Forge Day - January

- Quarterly Native Plant Sales

- $\quad$ Arbor Day Plant Sale (February)

- $\quad$ Spring Plant Sale (April)

- $\quad$ Aquatic Plant Sale (July)

- $\quad$ Fall Plant Sale (October)

- Wildlife Day (March)
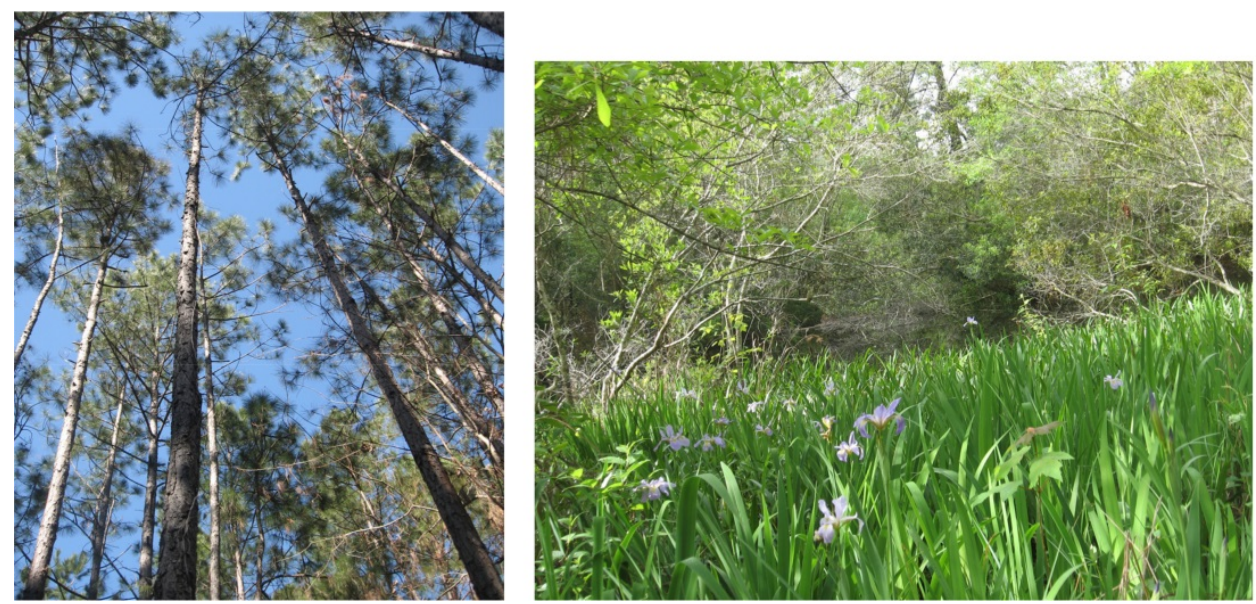

Figure 6. Some views from South Savanna Exhibit (Photos: Banu Ozturk Kurtaslan, 2011).

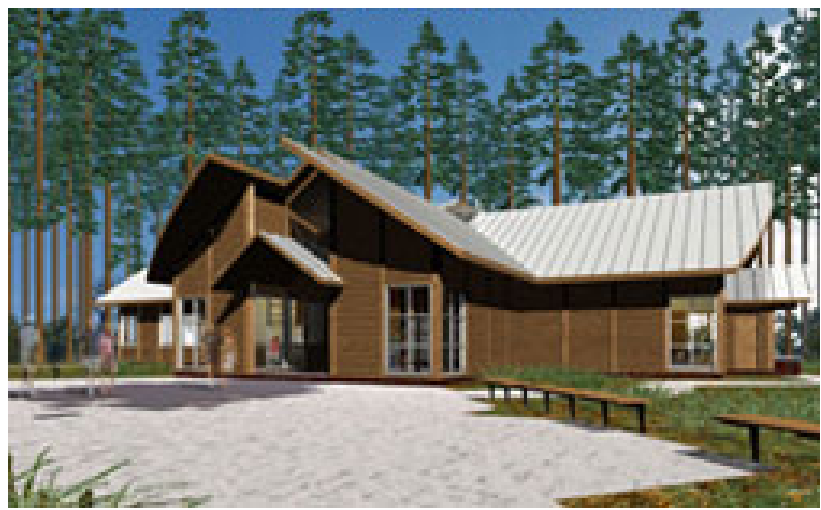

Figure 7. New Education Center building

(http://www.crosbyarboretum.msstate.edu/pages/visitors.php) 
- $\quad$ Strawberries and Cream - April

- Summer Nature Camp - June

- $\quad$ Bugfest - Third Weekend in September

- $\quad$ Piney Woods Heritage Festival - Second Weekend in November (Figure 8).

- $\quad$ Arboretum Open House - December

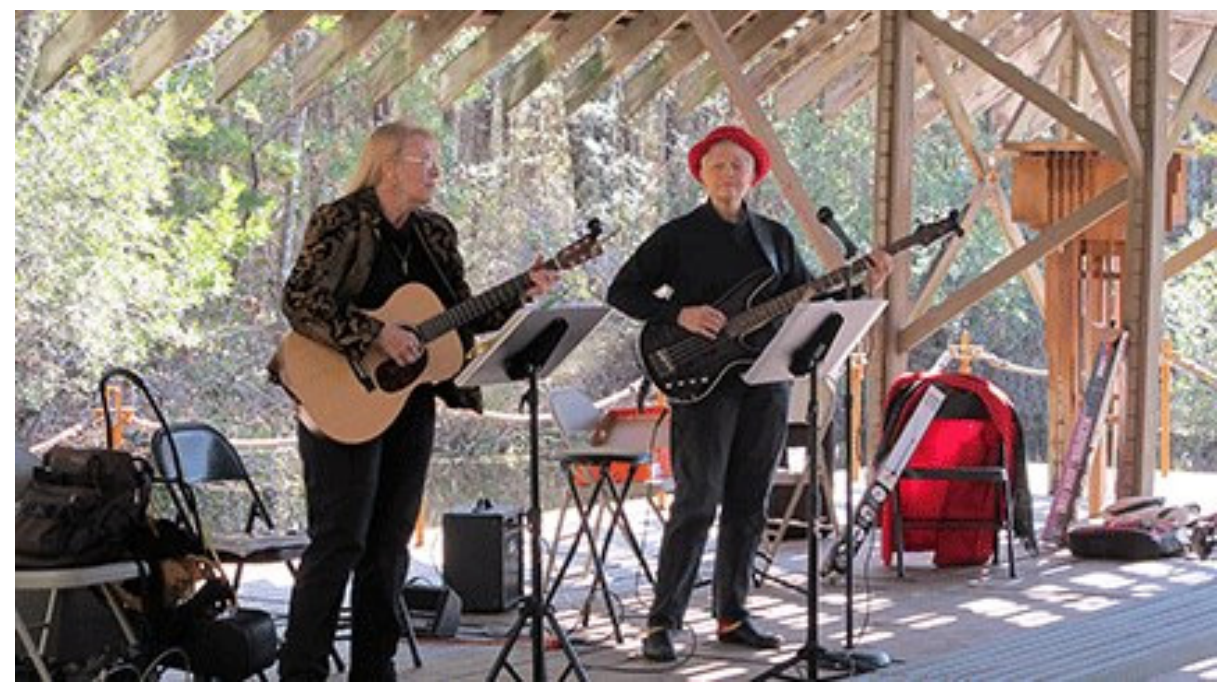

Figure 8. A concert as part of Piney Woods Heritage Festival

\section{WINTER 2012:}

- Winter sparrow banding field walk: A coastal bird biologist, MSU Coastal Research \& Extension Center, will conduct this field walk and workshop that will focus on mist netting winter sparrows (primarily Henslow's sparrows) and give participants an opportunity to observe and handle wild birds.

- Holiday ornaments for backyard wildlife-Kids: Birds and other critters often need extra food in the winter for fuel and warmth. Children will enjoy making tasty delights such as peanut butter pinecone feeders and popcorn-cranberry garlands that will attract birds and wildlife to your backyard.

- Arboretum open house

- Introduction to birding-family: An avid birder and writer, will discuss the fun aspects of birding, including feeding and identification tips. Learn about our resident birds and migrants that visit each year, books, equipment, materials, and other birding resources.

- Wild about winter: The advanced Project Wild workshop will focus on native Mississippi winter wildlife and how to incorporate seasonal wildlife, particularly migratory species and organisms' winter adaptations, into the classroom.

- Forge day: blacksmthing and metalworking: A chance to learn techniques and tips to get started in metalworking. The demonstrations, or try the hand at the forge (Figure 9)

- Arbor day native plant sale 
- Winter botany field walk - Family: With a Mississippi Department of Wildlife, Fisheries, \& Parks Botanist, in an exploration of the Pond and Savanna Habitats of the Crosby Arboretum.

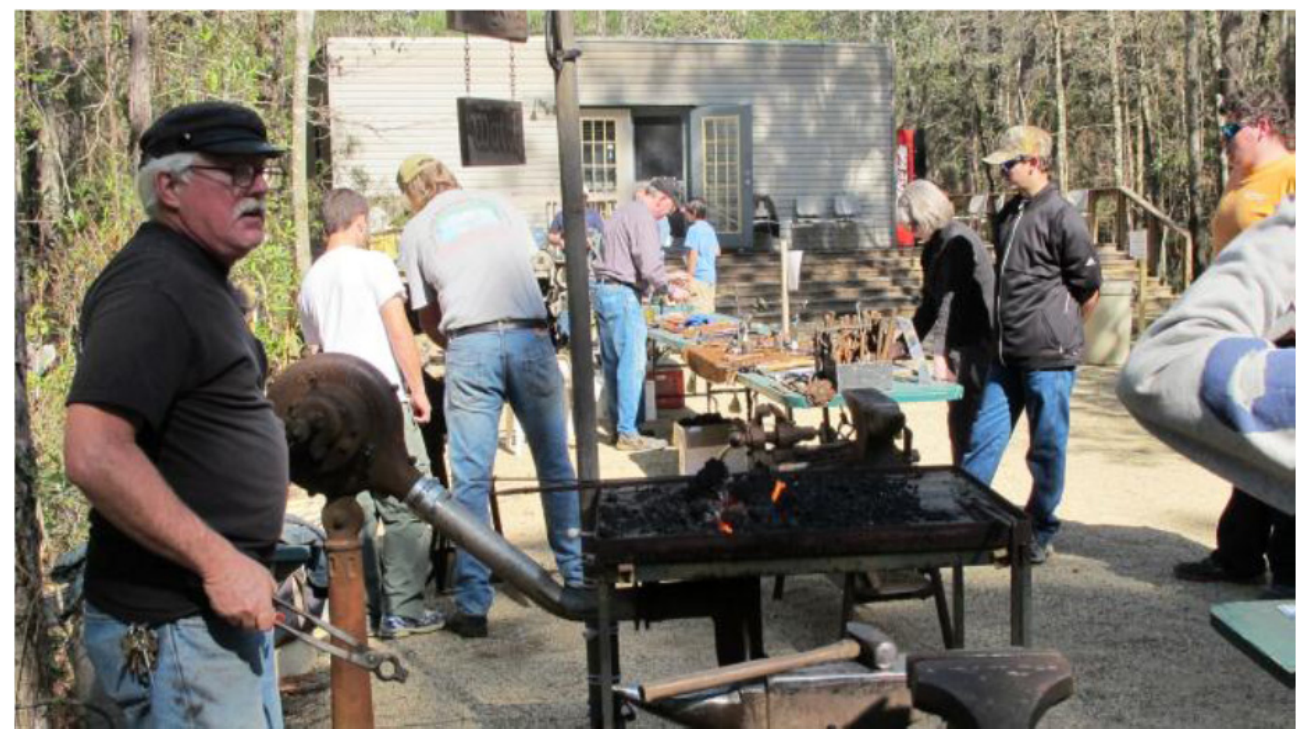

Figure 9. Forge day at the arboretum

\section{SPRING 2012:}

- "Flying wild": project wild teacher's workshop: Flying wild is designed to inspire young people to discover more about conservation and the natural world, and to get involved in activities that promote environmental learning and stewardship.

- Firewise event: "how to have a firewise home"- Adults: Information will be presented on how to design, construct, landscape, and maintain home or community so as to withstand a wildfire without the aid of firefighting resources on scene.

- Wildlife day-School Day-: Children will be thrilled to see the array of exhibitors displaying live and preserved animals, while learning how to protect and maintain wildlife in a field day open to area K-12 schools and homeschool groups.

- The Jean Chisholm Lindsey lecture in landscape design -Adults-: at the Welty House Garden featuring authors of One Writer's Garden: Eudora Welty's Home Place.

- Strawberries \& Cream festival: To celebrate the history of the old strawberry farm on the Pinecote Pavilion. Ice cream, fresh strawberries, and Picayune Frog Lemonade will be served.

- Spring plant sale

- $\quad$ Spider day- Family: 10:00 a.m. - 2:00 p.m. MSU Entomology Student will begin the day with a 30-minute tarantula presentation in the Pinecote Pavilion. A 30 minute talk on venomous spiders will be conducted. 
- Spring field walk: native plants for the home landscape: Join Director for a field walk through the Aquatic, Woodland, and Savanna Exhibits to discuss the plants growing in these habitats and how to use them in your home landscape.

- Earth day at the arboretum: A program, or visit exhibits that focus on nature and sustainable gardening. Programs: Earth day strategies used to protect Mississippi's coastal habitat, get to know native azaleas.

- Painted pots -kids: Decorating a clay pot and design a Mother's Day Card using recycled materials (Figure 10).

- The native orchids of south Mississippi - Adults: Learning to recognize and enjoy many of the thirty species of orchids native to the Gulf Coast.

- "Walking with Kim": A walk while enjoying the great outdoors.

\section{SUMMER 2012}

- Green Fire Film and Discussion: Viewing the new documentary, Green Fire: Aldo Leopold and a Land Ethic for Our Time. An extension Forestry Professor, will lead an interesting and lively discussion about the documentary from the perspective of "Aldo" Suited to ages 7 and up.

- Kid's summer nature camp: Children ages 6 through 12 will enjoy this four-day camp, learning about the outdoors with fun hands-on lessons, games, and activities.

- Teachers' workshop-wild about art and math: This interdisciplinary workshop is open to teachers and homeschool educators. A fun-filled, hands-on Project Wild workshop emphasizing art and math, conducted by Mississippi Museum of Natural Science Educational Outreach Biologist.

- Crosby arboretum's summer aquatic plant sale

- Hummingbirds: Understanding Ruby-throated Hummingbirdsin your yard: James Bell, Hummingbird Bander, of the Hummer / Bird Study Group Inc. will explain what is going on in the hummingbirds life, how they feed, why they fight so much, how to overcome that one bad bird that thinks he owns the feeder, simple tips and tricks that will insure you see more hummingbirds, and enjoy the beauty of one of nature's most dazzling creations.

- Teachers' workshop: wild About Creepy Crawlies: The Project Wild workshop will focus on native Mississippi "creepy crawlies" and will be conducted by Crystie Baker, Mississippi Museum of Natural Science Educational Outreach Biologist.

- Field walk \& clay class: Children will journey down the Arboretum pathways with director to collect natural materials they will use to impress into clay and form lasting memories.

- Summer arboretum field work: Trees, shrubs, and herbaceous plant material native to the region will be discussed, including uses in the home landscape.

FALL 2012

- Mushroom walk: Learning about fungi's fascinating ecology, taxonomy, and relationship to mankind with a professor in Biology from the University of South Alabama (Figure 11). 


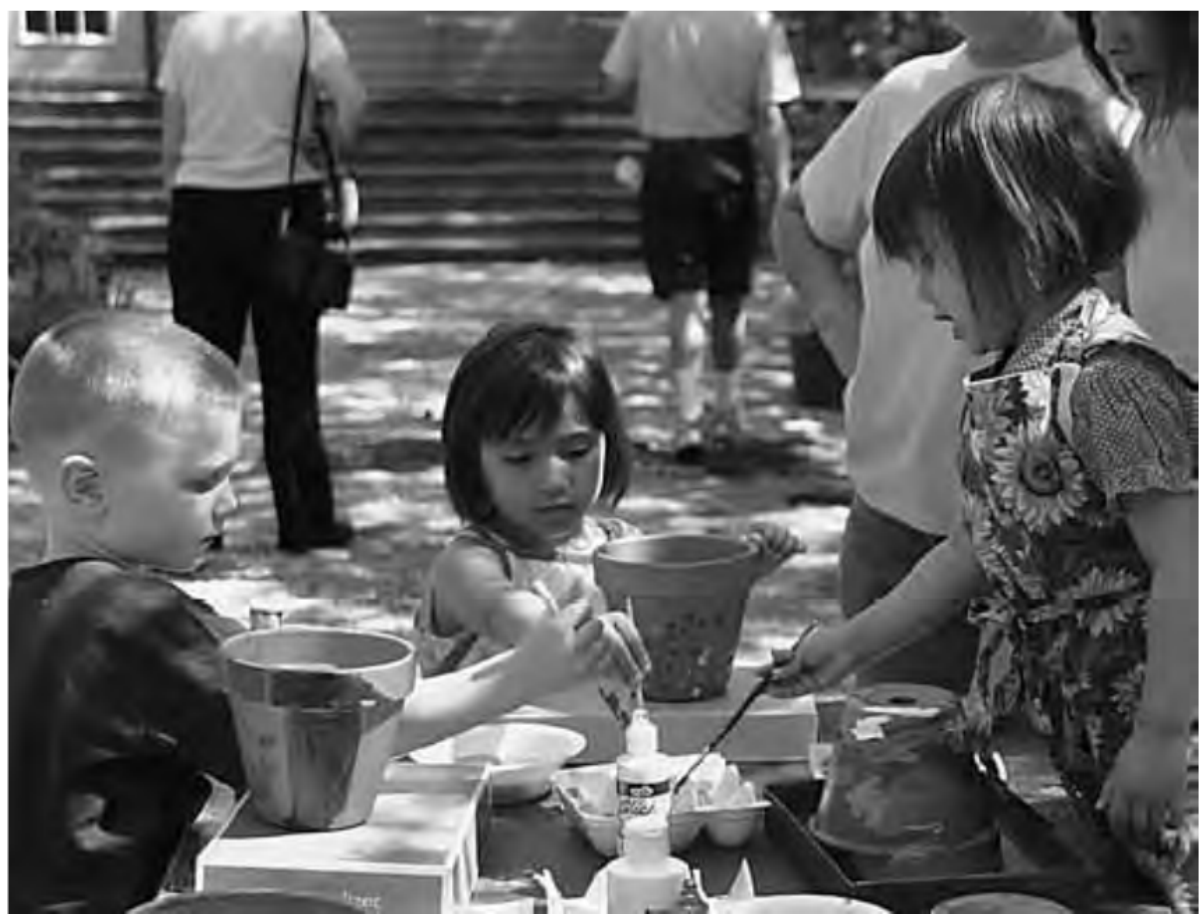

Figure 10. Decorating a clay pot for Mother's Day (http://www.crosbyarboretum.msstate.edu/pdf/2011\%20Crosby\%20Summer\%202011_WE Bsmall.pdf)

- Bugfest family event: Insect collecting by school and homeschool groups (groups over 20 persons must call to pre-register for an arrival time).

- Fall native plants sale

- Winter Sparrow Banding Field Walk

- Wildlife ornaments-Kids

- Arboretum Open House

- Project Learning Tree workshop: One of the oldest and most successful environmental education programs in the world. PLT activities are unbiased, interdisciplinary, fun, hands-on lesson plans, based on sound science. The goal is to "teach students how to think not what to think about environmental issues." For early childhood.

- The Annual Piney Woods Heritage Festival: Offers visitors a chance to see, hear, taste, learn, and participate in all sorts of crafts and activities from the Piney Woods region. Celebrating the early days of the Piney Woods in this 10th annual festival with exhibits and demonstrations of traditional skills such as blacksmithing, quilting, spinning, basket-making, and more.

- Learn the art of bonsai

- Painted Pumpkins - Kids

- Yoga at Pinecote Pavilion - Adults (Figure 12). 


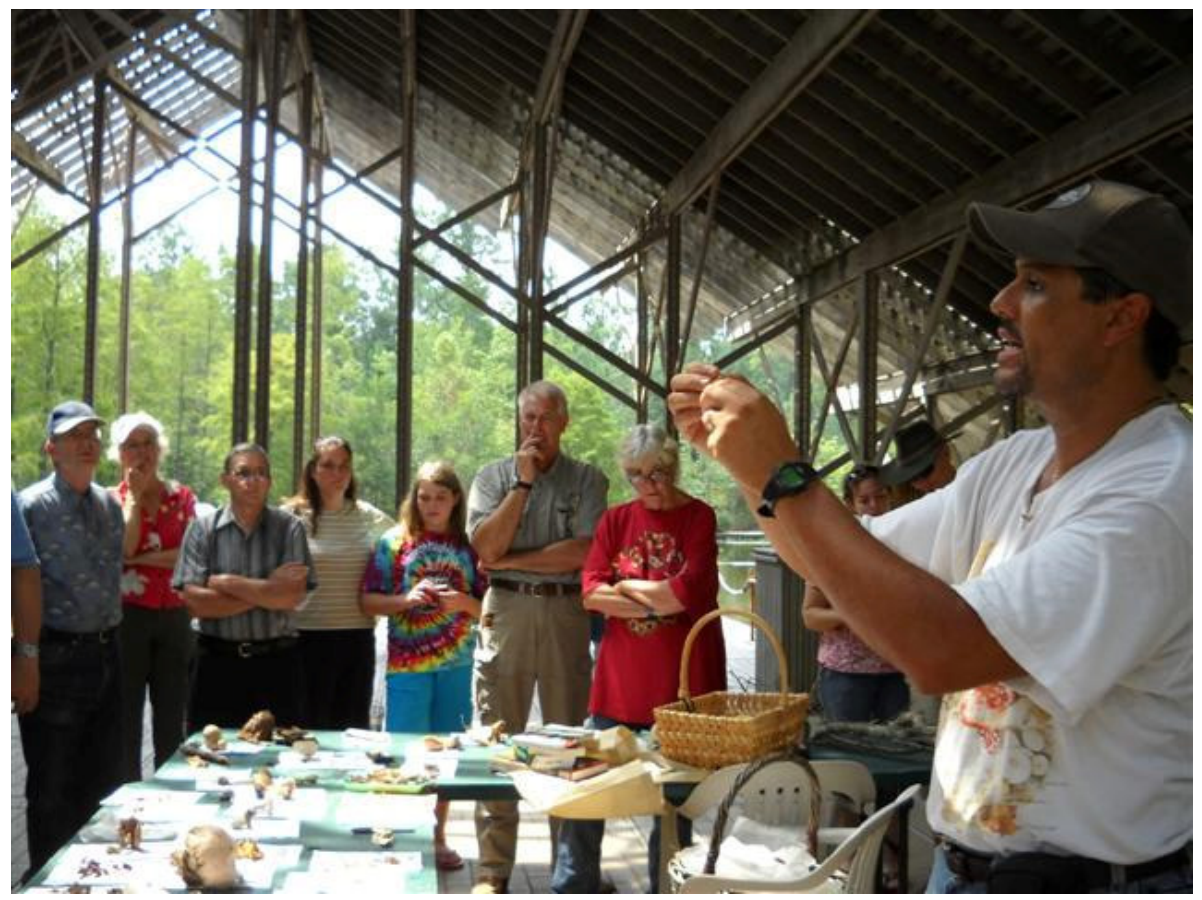

Figure 11. A wiew from "mushroom walk" activity

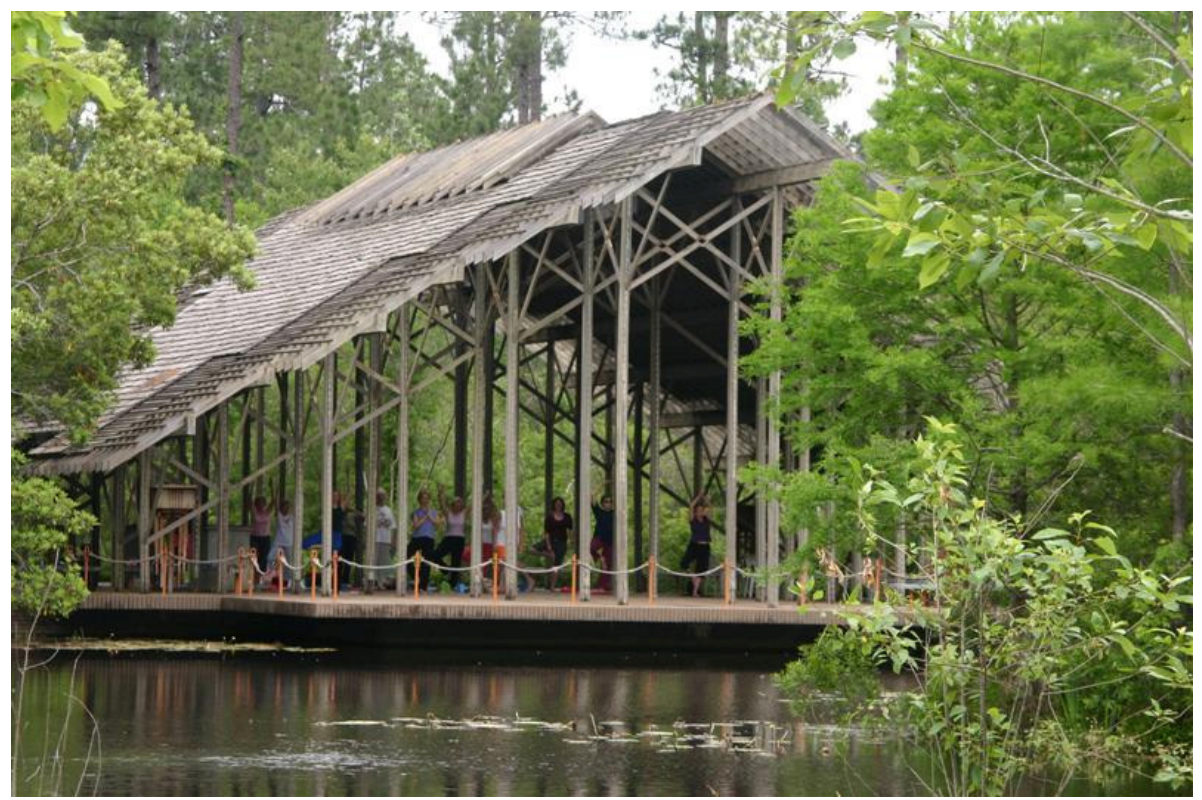

Figure 12. Yoga class at Pinecote Pavillion 
- $\quad$ Fall field walk: Native plants for the home landscape

- Girl scout environmental badges day: Scouting groups may picnic before or after program times on the grounds as part of their visit.

In addition to these activities and events, every Friday arboretum volunteers meet. Volunteering presents very important contributions for the arboretum (Figure 14).

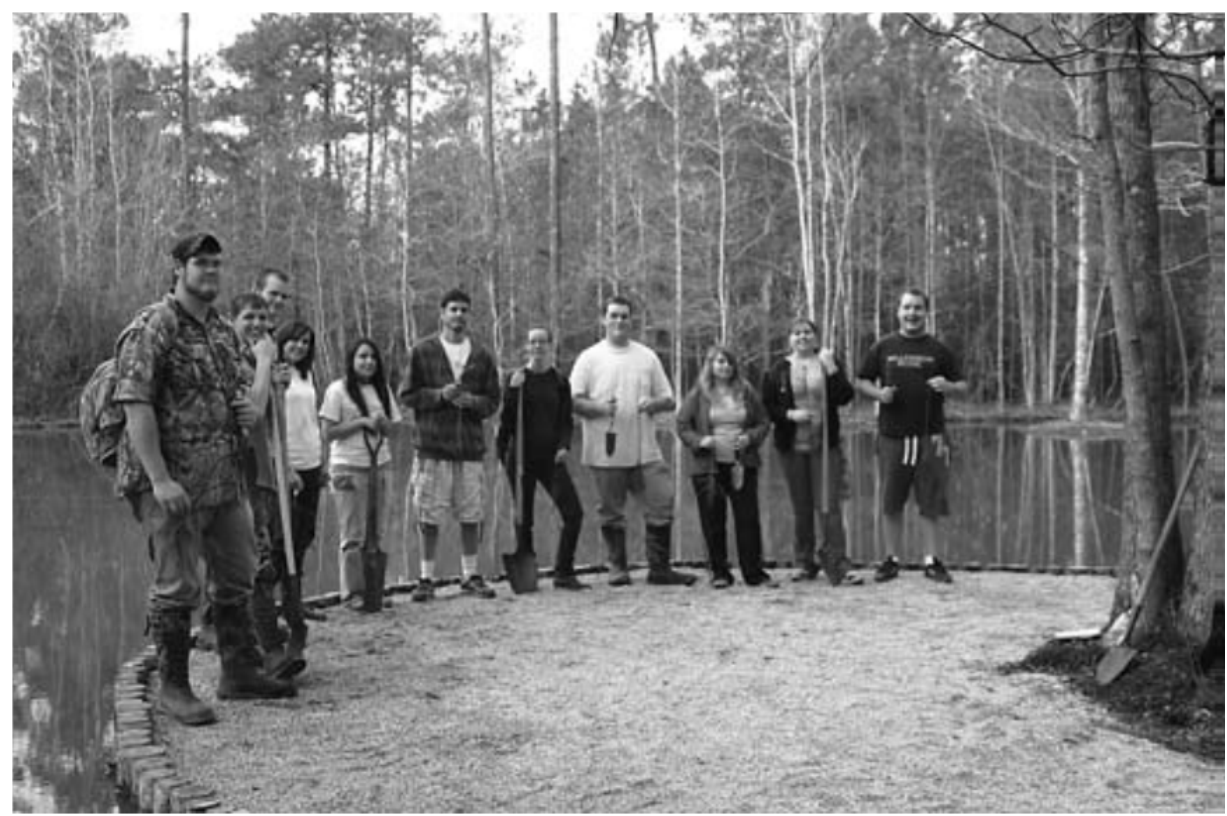

Figure 13. Joyce Applegate's Pearl River Community College botany class planted swamp gum trees on February 16, 2011 as volunteer

(http://www.crosbyarboretum.msstate.edu/pdf/2011\%20Crosby\%20Spring\%202011_Web.pdf)

\section{Conclusions}

Crosby Arboretum is a public garden that has constituted with the efforts of ecological design. The ecological, recreational and esthesic values has been attracting a number of people both from its own site and the world. Especially, Pinecote's aquatic displays serves visitors to Southern Mississippi a rich taste of the diversity of ife that resides here. The displays in the arboretum are designed to educate visitors with lots of activities and events. The goal of the arboretum staff is try to show the importance of these environmental processes both in natural areas and around all human development. So the unique sense of the place is preserved and celebrated with all other people. Crosby is fulfilling its missions of "preserving, protecting, and displaying plants native to the Pearl River Drainage Basin ecosystem, providing environmental and botanical research opportunities, and offering cultural, scientific, and recreational programs" with a succesful planninng, design and management. 


\section{Author details}

Banu Ozturk Kurtaslan

Selcuk University Faculty of Agriculture, Department of Landscape Architecture, Turkey

Robert Brzuszek

Mississippi State University, Department of Landscape Architeture, USA

\section{References}

[1] Yaltirik, F., 1969. Canli ve Kurutulmus Bitki Muzeleri (Arboretum, Botanik Bahcesi, Herbaryum), Istanbul Universitesi Orman Fakultesi Dergisi, Seri B, !9. Cilt, Sayi:1.

[2] Sertkaya, S. 1997. Bartin Orman Fakultesi Arboretumu'nun Kurulmasina Yönelik Bir Arastirma. Yüksek Lisans Tezi. Zonguldak Karaelmas Universitesi Peyzaj Mimarligi Anabilim Dali Zonguldak.

[3] Francis, J. K., 1989. The Luquillo Experimental Forest Arboretum. United States Department of Agricultry, Forest Service, Southern Forest Experiment Station, pp. 1-8. USA.

[4] Sat, B., 2002. Doga Koruma ve Cevre Egitimi Acisindan Arboretumlarin Islevleri ve Ataturk Arboretumu. Istanbul Universitesi Peyzaj Mimarligi Bolumu. Istanbul.

[5] McHarg, I., 1969. Design with Nature. Doubleday/Natural History Press, Garden City, NY.

[6] Mozingo, L. 1997. The Esthetics of Ecological Design: Seeing science as a culture. Landscape Journal. 16 (2): 46-59.

[7] Van der Ryan, S., and Cowan, S., 1996. Ecological Design. Island Pres. Washington D.C. 1996. pp. 200.

[8] Spirn, A.W., 1984. The Granite Garden: Urban Nature and Human Design. Basic Books, New York.

[9] Hough, M., 1995. Cities and Natural Process. Routledge, New York.

[10] Brzuszek, B. and Clark, J., 2009. The Crosby Arboretum. Native Plants. 10:2. Summer.

[11] Anonymus, 1984. Crosby Arboretum Quarterly News Journal. Vol. 2.1

[12] Brzuszek, B. 2012. Interview. The Curator of Pinecote, Crosby Arboretum. Mississippi State University Landscape Architecture Department. Starkville, USA.

[13] Anonymus 2011. Crosby Arboretum-The Small Stream Swamp Forest Exhibit Master Plan. Booklet by the graduate class in the department of landscape architecture at Mississippi State University. Starkville.

[14] Brzuszek, R., 2010. Artful Disturbance in The Piney Woods Landscape-The Crosby Arboretum.New Directions in American Landscape. Power Point Presentation. Connecticut College, Connecticut.

[15] Templeton, K. and Templeton, B., 2009. Crosby Arboretum: A Celebration of Nature. The Crosby Arboretum- Mississippi State University Extension Service. Quarterly News Journal. Spring 2009. pp. 8-9. USA. 
[16] Randall L. Meador, 2010. The Crosby Arboretum- Mississippi State University Extention Service. Quarterly News Journal. Spring.24.2. MSU Masters of Landscape Architecture student and civil engineer, Neel Schaffer, Inc.

[17] Anonymus, 2006. The Crosby Arboretum- Mississippi State University Extension Service. Quarterly News Journal. Spring 2006. pp. 223-229. USA.

[18] Brzuszek, R., 2011. Interview. The Curator of Pinecote, Crosby Arboretum. Mississippi State University Landscape Architecture Department. Starkville, USA.

[19] American Society of Landscape Architects, 1991. Landscape Architecture Magazine, November 1991.

[20] Mozingo, L., 1997. The Esthetics of Ecological Design: Seeing science as a culture. Landscape Journal. 16 (2): 46-59.

[21] Brzuszek, R., 1994. Celebrating Water at the Crosby Arboretum. Crosby Arboretum Quarterly News Journal. October 1994.

[22] Spirn, A.W., 1984. The Granite Garden: Urban Nature and Human Design. Basic Books, New York.

[23] Patchett,J. M. And Wilhelm, G.S. Conservation Design Forum, Inc. James M. Patchett \& Gerould S. Wilhelm. Conservation Design Forum, Inc. USA.

[24] Betz, S. 2004. Kids, Gardens and Art. A Natural Connection. The Herbarist. Issue 70.pp 45-48. 